Pacific Journal of Mathematics

SMOOTHNESS OF ANALYTIC FUNCTIONS AT BOUNDARY

MisKiTO HAYASH 


\section{SMOOTHNESS OF ANALYTIC FUNCTIONS AT BOUNDARY POINTS}

MIKIHIRO HAYASHI

Let $U$ be a bounded open set in the plane. We study the smoothness at boundary points of the continuous functions on $\bar{U}$ which are analytic in $U$. A main result is the characterization of sequences $x_{n} \in \bar{U}, x_{n} \rightarrow x$, with the property that the functions are of class $C^{k}$ along $x_{n}$ at $x$. As an application of this characterization, we can find an open set $U$ for any twice continuously differentiable arc $J$ such that $U$ contains $J$ in its boundary and the functions are of class $C^{\infty}$ on $J$.

1. Introduction. Let $U$ be a bounded open set in the complex plane. $\quad \bar{U}$ and $b U$ will be the closure and the boundary of $U$, respectively. $A(U)$ will be the set of all continuous functions on $\bar{U}$ which are analytic in $U$. The set $A(U)$ is a Banach space with the supnorm, $\|f\|=\|f\|_{U}=\sup \{|f(z)|: z \in U\}$.

For a point $x \in U$, the derivative $f^{(t)}(x)$ is regarded as a linear functional $f \mapsto f^{(t)}(x)$ on the Banach space $A(U)$. By Cauchy's integral formula this functional is bounded. For some boundary point $x$ of $U$, the derivative $f^{(t)}(x)$ is defined as a bounded linear functional on $A(U)$; A. P. Hallstrom [2] has characterized such points $x \in b U$ in terms of the analytic capacity (cf. Theorem 2.1). However, this does not mean that the functions in $A(U)$ are actually differentiable at $x$; in fact, we can construct an example such that all order derivatives $f^{(t)}(x)$ at a point $x$ are bounded functionals, while the first order derivative of a function in $A(U)$ is not continuous at $x$ along $x_{n}$ for any sequence $x_{n} \rightarrow x$ (Example $3, \S 3$ ). The purpose of this paper is to investigate the sequences $\left\{x_{n}\right\}$ along which the functions in $A(U)$ are actually differentiable at $x$.

The problem was first considered by A. G. O'Farrell [4], J. L. Wang [6] and the author [3]. The first and the second authors used suitable Borel measures as a main tool and considered $k$ th order differentiability, and so on. Independently, we have treated the same problem by using both the measures and the analytic capacity. However, in our previous paper we have considered only the first order case. Suggested by their papers we succeeded in the extension of the capacity's method for the high order case; for which we need an improvement of the method.

Our results are collected in the next section. A main result is Theorem 2.2; roughly speaking, the sequences $x_{n} \rightarrow x$ are characterized 
by the degree of thickness of the interior $U$ at $x$ and $x_{n}$. To measure the thickness, we shall use the analytic capacity. Applying this theorem, we shall construct some examples in $\S 3$. The main part of our proof consists of calculations to estimate the remainder of Taylor expansion in terms of the analytic capacity. These calculations will be made in $\S \S 4$ and 5 . In $\S 6$ we shall show a way of direct proof for the properties of $\alpha_{t}(x, r)$; this notation will be defined at the beginning of the next section.

The similar results are valid for the classical functions spaces $H^{\infty}(U)$ and $R(X) . \quad H^{\infty}(U)$ is the space of all bounded analytic functions on $U . R(X)$ is the space of all continuous functions on a compact plane set $X$ which are uniformly approximable by rational functions with no pole on $X$. The comments on these corresponding results will be made at suitable places of $\S 2$.

We wish to thank the referee for his variable suggestions and especially for showing us Lemma 2.10 in the present form so that we can carry out the proof of the theorem without the aid of $\S 6$. Our original form of Lemma 2.10 is stated as the statements (iv) and $(\mathrm{v})$ in $\S 6$.

2. Results and estimates. Throughout the paper, $U$ will be a bounded open set in the plane, and we shall fix a number $a$ with $0<a<1$. Under this convention, $E_{n}(x, r)=E_{n}(x, r, a)$ will denote the open annuli $\left\{z: r a^{n+1}<|z-x|<r a^{n}\right\}$ for integers $n,-\infty<n<\infty$. And the open disc $\{z:|z-x|<r\}$ will be denoted by $\Delta(x, r)$.

For a set $E$ in the complex plane $C$, let $\mathscr{C} \mathscr{C}(E)$ be the set of all continuous functions $f$ on the Riemann sphere $S^{2}=C \cup\{\infty\}$ such that $f$ is analytic outside of a compact subset of $E$ and $\|f\|_{S^{2}} \leqq 1$, $f(\infty)=0$. Then, the continuous analytic capacity, $\alpha(E)$, of the set $E$ is defined by

$$
\alpha(E)=\sup \left\{\left|f^{\prime}(\infty)\right|: f \in \mathscr{A} \mathscr{C}(E)\right\} .
$$

The following two facts are seen easily:

$$
\begin{gathered}
\alpha(\Delta(x, r))=r, \\
\alpha\left(E_{1}\right) \leqq\left(E_{2}\right) \text { if } \quad E_{1} \leqq E_{2} .
\end{gathered}
$$

To measure the thickness of the interior $U$ at $x$, we shall consider the following quantity:

$$
\alpha_{t}^{H, N}(x, r)=\alpha_{t}^{M, N}(x, r, a)_{U}=\frac{1}{(1-a) a^{t}} \sum_{n=M}^{N} \frac{\alpha\left(E_{n}(x, r) \backslash U\right)}{\left(r a^{n}\right)^{t}},
$$

where $t$ is a nonnegative integer and $M, N$ are a pair of integers 
with $-\infty<M \leqq N<\infty$ or $N=\infty$. Clearly, it follows that

$$
\alpha_{s}^{0, N}(x, r) \leqq r^{t-s} \alpha_{t}^{0, N}(x, r) \text { for } 0 \leqq s \leqq t .
$$

We shall use the notation $\alpha_{t}(x, r)$ instead of $\alpha_{t}^{0, \infty}(x, r)$. Some other abbreviations, such as $\alpha_{t}(x, r)_{U}$ and $\alpha_{t}(x, r, a)$, will be used if it is appropriate for the occasion. Note that this quantity measures the outside of $U$, so $U$ is thick at $x$ if it is small.

REMARK. To give a general form of estimates including the parameter $a$, we are working with general $a$. This, however, is a matter of interest but not of necessity. It may be helpful for the reader to regard $a=1 / 2$ in this paper, by which each expression will become simpler.

Now, let $x \in \bar{U}$. We shall denote by $A(U ; x)$ the set of the functions in $A(U)$ which admit analytic continuation to some neighborhood of $[x$. It is known that $A(U ; x)$ is a uniform dense subspace of $A(U)$ (cf. [1; Chap. II, Th. 1.8]). We define a linear functional on $A(U ; x)$ by

$$
D_{x}^{t}: f \longmapsto \frac{1}{t !} f^{(t)}(x) .
$$

If the norm $\left\|D_{x}^{t}\right\|$ is bounded, then we can extend $\left\|D_{x}^{t}\right\|$ uniquely to a bounded linear functional on $A(U)$; in this case, we may use the notation $f^{(t)}(x)=t$ ! $D_{x}^{t} f$ for all $f \in A(U)$.

In our notations Hallstrom's characterization reads as follows:

Theorem 2.1 ([2]). Let $x \in U$ and let $r>0$. Then $D_{x}^{t}(t \geqq 1)$ is bounded if and only if $\alpha_{t+1}(x, r)<\infty$.

Since the functional $D_{x}^{t}$ is defined by $A(U), x$ and $t$, the finiteness of $\alpha_{t}(x, r, a)$ does not depend on numbers $r$ and $a$. Now, our main theorem reads as follows:

TheOREM 2.2. Let $x \in \bar{U}$ and $x_{n} \in \bar{U}$ be a sequence converging to $x$.

(i) Suppose $\alpha_{t+1}(x, r)<\infty(t \geqq 1)$. Then

$$
f^{(t)}\left(x_{n}\right) \longrightarrow f^{(t)}(x)
$$

for all $f \in A(U)$ (resp., uniformly for $f \in A(U),\|f\| \leqq 1$ ) if and only if

$$
\left.\varlimsup_{n \rightarrow \infty} \alpha_{t+1}\left(x_{n}, \rho\left|x_{n}-x\right|\right)<\infty \quad \text { (resp., } \lim _{n \rightarrow \infty}=0\right) .
$$


Here, $\rho>0$ is any fixed number.

(ii) Suppose $\alpha_{t+2}(x, r)<\infty(t \geqq 0)$. Then

$$
\frac{f^{(t)}\left(x_{n}\right)-f^{(t)}(x)}{x_{n}-x} \longrightarrow f^{(t+1)}(x)
$$

for all $f \in A(U)$ (resp., uniformly for $f \in A(U),\|f\| \leqq 1$ ) if and only if

$$
\left.\varlimsup_{n \rightarrow \infty} \frac{\alpha_{t+1}\left(x_{n}, \rho\left|x_{n}-x\right|\right)}{\left|x_{n}-x\right|}<\infty \quad \text { (resp., } \lim _{n \rightarrow \infty}=0\right) .
$$

Here, $\rho>0$ is any fixed number.

Note. We proved in our previous paper [3] that the analogue in case $t=0$ of the uniform convergence part of Theorem 2.2 (i) is valid. However, the pointwise convergence part is false in case $t=0$. For a counterexample, let $x$ be a nonpeak point on the boundary of $U$ to which a sequence $x_{n}$ of peak points converges. Then $\alpha_{1}(x, r)<\infty$ and $\alpha_{1}\left(x_{n},\left|x_{n}-x\right|\right)=\infty$ for all $n$, but $f\left(x_{n}\right) \rightarrow f(x)$ for all $f \in A(U)$.

Theorem 2.1 is the consequence of the following estimates:

Estimate 2.3 (cf. (4.4), (5.2)). Let $x \in \bar{U}$ and

(i) Then, it holds for $r>0$

$$
\left\|D_{x}^{t}\right\| \geqq C_{1} \sup _{N} \frac{\alpha_{t+1}^{0, N}(x, r)}{\alpha_{1}^{0, N}(x, r)+3},
$$

where $C_{1}=C(a, t)$ is an absolute positive constant which depends only on a and $t$.

(ii) If $R$ is a positive number with $\bar{U} \subseteq \Delta(x, R)$, then it holds

$$
\left\|D_{x}^{t}\right\| \leqq c_{0} \alpha_{t+1}(x, R),
$$

where $c_{0}$ is a universal constant.

Later, many positive constants, as $C_{1}$, will appear. They will depend on several parameters $*_{1}, \cdots, *_{p}$ and do not depend on $U$ and $x$. To describe such constants, we shall say that $C=C\left(*_{1}, \cdots, *_{p}\right)$ is an absolute constant. A universal constant is an absolute constant with no parameter; in the paper, however, we shall not use a universal constant other than $c_{0}$, which is the constant that appears in Melnikov's estimate (cf. $\S 5,(5.1)$ ); from now on we shall use this constant $c_{0}$ without notice.

Some detail points should be mentioned for the proof of Theorem 2.1. Since $\alpha_{1}^{0, N}(x, r) \leqq r^{t} \alpha_{t+1}^{0, N}(x, r)$ by (2.3), the estimate (E-1) implies 


$$
\alpha_{t+1}(x, r) \leqq \frac{3\left\|D_{x}^{t}\right\|}{C_{1}-r^{t}\left\|D_{x}^{t}\right\|}
$$

if $r<\sqrt[t]{C_{1} /\left\|D_{x}^{t}\right\|}$. Therefore, we need the fact that $\alpha_{t}(x, r)<\infty$ for some $r>0$ then $\alpha_{t}\left(x, r^{\prime}\right)<\infty$ for a sufficiently small (or large) number $r^{\prime}>0$; to see this, we have only to put $r^{\prime}=r a^{N}$ in the equality $\alpha_{t}\left(x, r a^{N}\right)=\alpha_{t}^{N, \infty}(x, r)$.

Note. The proof of the estimates (E-1) and (E-1') are essentially due to A. P. Hallstrom [2]. He also showed that if $\alpha_{1}(x, r)=\infty$ then $\lim _{N \rightarrow \infty} \alpha_{t+1}^{0, N}(x, r) / \alpha_{1}^{0, N}(x, r)=\infty$ for $t \geqq 1$. This follows from Cauchy's inequality

$$
\begin{aligned}
\left\{\alpha_{1}^{0, N}(x, r)\right\}^{2} & \leqq \alpha_{t+1}^{0, N}(x, r) \sum_{n=0}^{N} \frac{\alpha\left(E_{n}(x, r) \backslash U\right)}{\left(r a^{n}\right)^{1-t}} \\
& \leqq \frac{r^{t}}{1-a^{-t}} \alpha_{t+1}(x, r) .
\end{aligned}
$$

The last inequality follows from $\alpha\left(E_{n}(x, r) \backslash U\right) \leqq r a^{n} \quad((2.1)$, (2.2)). Therefore, the limit

$$
\lim _{N \rightarrow \infty} \frac{\alpha_{t+1}^{0, N}(x, r)}{\alpha_{1}^{0, N}(x, r)+3}=\frac{\alpha_{t+1}(x, r)}{\alpha_{1}(x, r)+3}
$$

exists, and is $+\infty$ if $\alpha_{1}(x, r)=\infty$. We shall not need this fact, however.

By (2.3) we have the following:

Corollary 2.4. If $D_{x}^{t}$ is bounded, then $D_{x}^{k}, k \leqq t$, are bounded.

Later, we shall need that following:

Lemma 2.5 (A. P. Hallstrom [2]). Let $x \in \bar{U}$. Suppose $\alpha_{t}(x, r)<\infty$. Then

$$
\lim _{r \rightarrow 0} \frac{\alpha(\Delta(x, r) \backslash U)}{r^{t}}=0
$$

A proof of this lemma will be given in $\S 6$.

To prove Theorem 2.2, we shall be concerned with the remainder of Taylor expansion: For $f \in A(U ; x)$, let

$$
\begin{aligned}
\left(R_{x}^{t} f\right)(z) & =f(z)-\sum_{k=0}^{t} \frac{(z-x)^{k}}{k !} f^{(t)}(x) \\
& =f(z)-\sum_{k=0}^{t}(z-x)^{k} D_{x}^{k} f .
\end{aligned}
$$


Clearly, $R_{x}^{t}$ is a linear operator on $A(U ; x)$. Since

$$
\left\|R_{x}^{t} f\right\| \leqq\|f\|\left(1+\sum_{k=0}^{t}\|z-x\|^{k}\left\|D_{x}^{k}\right\|\right),
$$

where $\|z-x\|=\sup _{z \in U}|z-x|, R_{x}^{t}$ is bounded provided $D_{x}^{t}$ is bounded; in this case, $R_{x}^{t}$ is uniquely extended to a bounded operator on $A(U)$ and it will be denoted by the same notation, $R_{x}^{t}$. Moreover, while the function $(1 / s !) f^{(s)}(z)$ is undefinable on the set $\bar{U}$, we can consider the Taylor expansion of that function: For $x, y \in \bar{U}$, we shall denote by $A(U ; x, y)$ the set of the functions in $A(U)$ which admit analytic continuation to some neighborhood of $x$ and $y$. The set $A(U ; x, y)$ is also a dense subspace of $A(U)$. Now, for $f \in A(U ; x, y)$, the remainder of Taylor expansion at $x$ of the function $(1 / s !) f^{(s)}(z)$ is given by $D_{y}^{s}\left(R_{x}^{t} f\right)$, for

$$
\begin{aligned}
D_{y}^{s}\left(R_{x}^{t} f\right) & =\frac{f^{(s)}}{s !}(y)-\sum_{k=s}^{t} \frac{(y-x)^{k-s}}{(y-s) !} \frac{f^{(k)}}{s !}(x) \\
& =D_{y}^{s} f-\sum_{k=s}^{t}\left(\begin{array}{c}
k \\
s
\end{array}\right)(y-x)^{k-s} D_{x}^{k} f .
\end{aligned}
$$

Our estimate of the norm $\left\|D_{y}^{s} R_{x}^{t}\right\|$ is as follows:

ESTimate 2.6 (cf. (4.5), (5.5)). Let $x, y \in \bar{U}, x \neq y$.

(i) If $0 \leqq s \leqq t$ and $0<\sigma<1$, then it holds

$$
\begin{aligned}
\left\|D_{y}^{s} R_{x}^{t}\right\| \geqq & \sup _{N} \frac{C_{2}}{\alpha_{1}^{0, N}(y, \sigma|y-x|)+C_{3}}\left(\alpha_{s+1}^{0, N}(y, \sigma|y-x|)\right. \\
& \left.-\sum_{k=1}^{s} \frac{3(k+1)}{a^{k}}\left(\begin{array}{c}
t+1 \\
k
\end{array}\right) \frac{\alpha_{s-k+1}^{0, N}(y, \sigma|y-x|)}{|y-x|^{k}}\right),
\end{aligned}
$$

where $C_{2}=C(a, t, s)$ and $C_{3}=C(a, t)$ are absolute constants.

(ii) If $0 \leqq s \leqq t, 0<\sigma<1$ and $|y-x| \leqq R / 4^{s+1}$, where $\bar{U} \leqq$ $\Delta(x, R)$, then it holds

$$
\begin{aligned}
\left\|D_{y}^{s} R_{x}^{t}\right\| \leqq & |y-x|^{t-s} \| R_{y}^{s}||\left(C_{4} \sqrt{\frac{|y-x|}{R}} \alpha_{t+1}(x, R)\right. \\
& \left.+C_{5} \alpha_{t+1}^{L, \infty}(x, R)+C_{6} \sum_{k=0}\left(\begin{array}{c}
t+1 \\
k
\end{array}\right) \frac{\alpha_{s-k+1}(y, \sigma|y-x|)}{|y-x|^{t-s+k}}\right),
\end{aligned}
$$

where $C_{4}=C(a, t, s), C_{5}=C(a, \sigma, t, s)$ and $C_{6}=C(a, \sigma, t)$ are absolute constants and $L=\left[1 / 2 \log _{a}|y-x| / R\right]$ (Gaussian symbol).

If $s=0$, then $D_{y}^{0}\left(R^{t} f\right)=\left(R_{x}^{t} f\right)(y)$ and $\left\|R_{y}^{0}\right\| \leqq 2$. In this case we have the following estimates.

Estimate 2.7. Let $x, y \in \bar{U}, x \neq y$, and $f \in A(U)$. 
(i) If $0<\sigma<1$, then it holds

$(\mathrm{E}-3)$

$$
\left|\left(R_{x}^{t} f\right)(y)\right| \geqq \frac{(1-a) a}{4 \cdot 2^{t}}\|f\| \frac{\alpha_{1}(y, \sigma|y-x|)}{\alpha_{1}(y, \sigma|y-x|)+\frac{6}{1-a}} .
$$
holds

(ii) If $0<\sigma<1$ and $|y-x| \leqq R / 4$, where $\bar{U} \subseteq \Delta(x, R)$, then it $\left(\mathrm{E}-3^{\prime}\right)$

$$
\begin{aligned}
\left|\left(R_{x}^{t} f\right)(y)\right| \leqq & \frac{3 c_{0}}{1-a}|y-x|^{t}\|f\|\left(\frac{4}{a} \sqrt{\frac{|y-x|}{R}} \alpha_{t+1}(x, R)\right. \\
& \left.+\frac{2}{\sigma^{2} a^{3}} \alpha_{t+1}^{L, \infty}(x, R)+\left(\frac{1}{1-\sigma}\right)^{t+1} \frac{a_{1}(y, \sigma|y-x|)}{|y-x|^{t}}\right),
\end{aligned}
$$

where $L=\left[1 / 2 \log _{a}|y-x| / R\right]$.

Note. We stated Estimate 2.7 as a special case of Estimate 2.6. However, we can prove the estimate $\left(\mathrm{E}-3^{\prime}\right)$ directly by modifying the calculation made in [3], which is rather more simple than that made in $\S 5$.

Now, we put the statement of Theorem 2.2 into a different form. As we have already noted, the condition $\alpha_{t+1}(x, r)<\infty$ is equivalent to the boundedness of the linear functional $D_{x}^{t}$ on $A(U)$. And, the condition " $f^{(t)}\left(x_{n}\right) \rightarrow f^{(t)}(x)$ for all $f \in A(U)$ (resp., uniformly for $f \in A(U),\|f\| \leqq 1$ )" is equivalent to the condition that $D_{x_{n}}^{t}$ converges weak-* (resp., in norm) to $D_{x}^{t}$. Moreover, the weak-* convergence of $D_{x_{n}}^{t}$ is equivalent to the condition $\overline{\lim }_{n}\left\|D_{x}^{t}\right\|<\infty$ in our case: The necessity of the latter condition is obvious by the uniform boundedness principle and, since $f^{(t)}\left(x_{n}\right) \rightarrow f^{(t)}(x)$ for $f \in A(U ; x)$, the sufficiency is a consequence of the following easy lemma.

Lemma 2.8. Let $E$ be a normed space and $E_{0}$ be a dense subset of E. If $\phi_{n}$ is a sequence of linear functionals on $E$ such that $\varlimsup_{\lim _{n}}\left\|\phi_{n}\right\|<\infty$ and $\phi_{n}(x)$ is converging for any $x \in E_{0}$, then $\phi_{n}$ converges weak-* to a bounded linear functional on $E$.

Now, since $D_{y}^{t}\left(R_{x}^{t} f\right)=D_{y}^{t} f-D_{x}^{t} f$, and since $\lim _{n} D_{x_{n}}^{t}\left(R_{x}^{t} f\right)=0$ for $f \in A(U ; x)$, the statement of Theorem 2.2, (i) is equivalent to the following: Let $\rho>0$ by any fixed number.

( i )' Suppose $\left\|D_{x}^{t}\right\|<\infty(t \geqq 1)$. Then,

$$
\varlimsup_{n}\left\|D_{x_{n}}^{t} R_{x}^{t}\right\|<\infty \quad \text { (resp., } \lim _{n}=0 \text { ) }
$$

if and only if

$$
\left.\varlimsup_{n} \alpha_{t+1}\left(x_{n}, \rho\left|x_{n}-x\right|\right)<\infty \quad \text { (resp., } \lim _{n}=0\right) .
$$


The same is good of the statement of Theorem 2.2, (ii). In this case, it holds that $D_{y}^{t}\left(R_{x}^{t+1} f\right)=D_{y}^{t} f-D_{x}^{t} f-(t+1)(y-x) D_{x}^{t+} f$. Hence, the statement of Theorem 2.2, (ii) is equivalent to the following: Let $\rho>0$ be fixed number.

(ii) Suppose $\left\|D_{x}^{t+1}\right\|<\infty(t \geqq 0)$. Then,

$$
\left.\varlimsup_{n} \frac{\left\|D_{x_{n}}^{t} R_{x}^{t+1}\right\|}{\left|x_{n}-x\right|}<\infty \quad \text { (resp., } \lim _{n}=0\right)
$$

if and only if

$$
\varlimsup_{n} \frac{\alpha_{t+1}\left(x_{n}, \rho\left|x_{n}-x\right|\right)}{\left|x_{n}-x\right|}<\infty \quad\left(\text { resp., } \lim _{n}=0\right) .
$$

Thus, Theorem 2.2 is special case of the following theorem.

THEOREM 2.9. Let $x \in \bar{U}$ and let $x_{n} \in \bar{U}$ be a sequence converging to $x$. Suppose $\left\|D_{x}^{t}\right\|<\infty$. Then, for $s \leqq t(t \geqq 1)$,

$$
\varlimsup_{n \rightarrow \infty} \frac{\left\|D_{x_{n}}^{s} R_{x}^{t}\right\|}{\left|x_{n}-x\right|^{t-s}}<\infty \quad \text { (resp., (2) } \lim _{n \rightarrow \infty}=0 \text { ) }
$$

if and only if

$$
\left.\varlimsup_{n \rightarrow \infty} \frac{\alpha_{s+1}\left(x_{n}, \rho\left|x_{n}-x\right|\right)}{\left|x_{n}-x\right|^{t-s}}<\infty \quad \text { (resp., (4) } \lim _{n \rightarrow \infty}=0\right) .
$$

Here, $\rho>0$ is any fixed number.

To prove the theorem, we need a lemma.

Lemma 2.10. Let $x, x_{n} \in \bar{U}, x_{n} \rightarrow x$, and let $\rho>0$. Suppose $\alpha_{t+1}(x, R)<\infty$.

(i) Let $s, k$ be nonnegative integers with $s+k \leqq t$. Then, it holds

$$
\lim _{n} \frac{\alpha_{s}^{M, N}\left(x_{n}, \rho\left|x_{n}-x\right|\right)}{\left|x_{n}-x\right|^{k}}=0
$$

for any integers $M, N$ with $M \leqq N$.

(ii) Let $N$ be an integer and, for each $n$, let $M(n)$ be an integer with $M(n) \leqq N$ and $a^{M(n)} \rho\left|x_{n}-x\right| \leqq R-\left|x_{n}-x\right|$. Then, it holds

$$
\varlimsup_{n} \alpha_{t}^{M(n), N}\left(x_{n}, \rho\left|x_{n}-x\right|\right) \leqq K,
$$

where $K$ is a constant independent of the sequences $x_{n}$ and the integers $N$ and $M(n)$. 
Proof. For simplicity, put $d_{n}=\left|x_{n}-x\right|$.

(i ) Since $E_{k}\left(x_{n}, \rho d_{n}\right) \cong \Delta\left(x, \rho d_{n} a^{M}\right)$ for $k \geqq M$, we have by (2.2)

$$
\begin{aligned}
\alpha_{s}^{M, N}\left(x_{n}, \rho d_{n}\right) & =\frac{1}{(1-a) a^{s}} \sum_{k=M}^{N} \frac{\alpha\left(E_{k}\left(x_{n}, \rho d_{n}\right) \backslash U\right)}{\left(\rho d_{n} a^{k}\right)^{s}} \\
& \leqq \frac{N-M+1}{(1-a) a^{s}} \frac{\alpha\left(\Delta\left(x, \rho d_{n} a^{M}\right) \backslash U\right)}{\left(\rho d_{n} a^{N}\right)^{s}} .
\end{aligned}
$$

Thus, Lemma 2.5 implies (i).

(ii) For a bounded set $E$ in the plane, put $r_{x}(E)=\inf \{r / R$ : $\{z: r<|z-x|<R\} \supseteqq E\}$. Thanks to (i), we may replace the integer $N$ to another one. So we assume

$$
r_{x}\left(E_{k}\left(x_{n}, \rho d_{n}\right)\right)=\frac{a^{k+1}-1}{a^{k}-1}<b=a^{3 / 2} \text { for } \quad k \leqq N(<0) .
$$

If $r_{x}(E)<b$, then the set $E$ can be covered by two succesive annuli $E_{m}(x, R, b)$ and $E_{m+1}(x, R, b)$. Putting $F_{m}=E_{m}\left(x, R, b^{2}\right)$ and $G_{m}=$ $E_{m}\left(x, b R, b^{2}\right)$, we have $E_{k}\left(x_{n}, \rho d_{n}\right) \subseteq F_{m}$, or $\subseteq G_{m}$ for $k \leqq N$, and hence,

$$
\frac{\alpha\left(E_{k l}\left(x_{n}, \rho d_{n}\right) \backslash U\right)}{\left(\rho d_{n} a^{k}\right)^{t}} \leqq \frac{1}{b^{2 t}} \frac{\alpha\left(F_{m} \backslash U\right)}{\left(R b^{2 m}\right)^{t}}, \quad \text { or } \leqq \frac{1}{b^{2 t}} \frac{\alpha\left(G_{m} \backslash U\right)}{\left(R b^{2 m-1}\right)^{t}} .
$$

Since $r_{x}\left(\bigcup_{i=0}^{2} E_{k+i}\left(x_{n}, \rho d_{n}\right)\right)>a^{3}$, each $F_{m}$ or $G_{m}$ does not contain more than two of $E_{k}\left(x_{n}, \rho d_{n}\right)$. Thus, when we sum up the above inequality about $k$, each $F_{m}$ or $G_{m}$ appears at most two times; and it holds

$$
\alpha_{t}^{M(n), N}\left(x_{n}, \rho d_{n}\right) \leqq \frac{1}{b^{2 t}}\left(\alpha_{t}\left(x, R, b^{2}\right)+\alpha_{t}\left(x, b R, b^{2}\right)\right) .
$$

This proves (ii).

REMARK. We can avoid the use of the fact $\alpha_{t}\left(x, \cdot, b^{2}\right)<\infty$ for a different conformal radius using the semi-additivity of the analytic capacity, we have $\alpha\left(E_{m}\left(x, R, \alpha^{3}\right) \backslash U\right) \leqq c_{0} /(1-a) \sum_{i=0}^{2} \alpha\left(E_{3 m+i}(x, R, a) \backslash U\right)$. Therefore, an easy calculation shows that

$$
\alpha_{t}^{M(n), N}\left(x_{n}, \rho d_{n}\right) \leqq \frac{12 c_{0}}{(1-a) a^{3 t+2}} \alpha_{t}(x, R) .
$$

Proof of Theorem 2.9. First, we assume that $\rho>0$ is so small that $\lambda=1-\sum_{k=1}^{s} 3(k+1)\left(\begin{array}{c}t+1 \\ k\end{array}\right)(\rho / a)^{k}>0$. Since $\alpha_{1}^{0, N}(y, \rho|y-x|) \leqq$ $\rho^{s}|y-x|^{s} \alpha_{s+1}^{0, N}(y, \rho|y-x|)$ and

$$
\alpha_{s-k+1}(y, \rho|y-x|) \leqq \rho^{k}|y-x|^{k} \alpha_{s+1}^{0, N}(y, \rho|y-x|)
$$


by (2.3), we have by (E-2)

$$
\alpha_{s+1}(y, \rho|y-x|) \leqq \frac{C_{3}\left\|D_{y}^{s} R_{x}^{t}\right\|}{\lambda C_{2}-\rho^{s}|y-x|^{s}\left\|D_{y}^{s} R_{x}^{t}\right\|},
$$

whenever the denominator is positive. Therefore, if $\lim _{n}\left\|D_{x_{n}}^{s} R_{x}^{t}\right\|=0$, then the property (1) (resp., (2)) implies the property (3) (resp., (4)). So, to complete the proof of the "only if" part, we have only to show that $\varlimsup_{\lim _{n}}\left\|D_{x_{n}}^{t} R_{x}^{t}\right\|<\infty$ implies

$$
\varlimsup_{n} \alpha_{s+1}\left(x_{n}, \rho\left|x_{n}-x\right|\right)<\infty .
$$

In fact, since $D_{x_{n}}^{t} R_{x}^{t}=D_{x_{n}}^{t}-D_{x}^{t}$ and $\left\|D_{x}^{t}\right\|<\infty$, the hypothesis implies $\varlimsup_{\lim _{n}}\left\|D_{x_{n}}^{t}\right\|<\infty$, and this implies $\varlimsup_{\lim _{n}} \alpha_{s+1}\left(x_{n}, \rho\left|x_{n}-x\right|\right)<\infty$ by the inequality (2.4).

Conversely, suppose that the property (3) (resp., (4)) holds. Then we have by (2.3)

$$
\left.\varlimsup_{n} \frac{\alpha_{s-k+1}\left(x_{n}, \rho\left|x_{n}-x\right|\right)}{\left|x_{n}-x\right|^{t-s+k}}<\infty \quad \text { (resp., } \lim _{n}=0\right)
$$

for $0 \leqq k \leqq s$. If it were proved that $\overline{\lim }_{n}\left\|R_{x_{n}}^{s}\right\|<\infty$, the property (1) (resp., (2)) would follow from the estimate $\left(\mathrm{E}-2^{\prime}\right)$. So we shall prove it. In either case, it follows that $\varlimsup_{n} \alpha_{s-k+1}\left(x_{n}, \rho\left|x_{n}-x\right|\right)<\infty$ for $0 \leqq k \leqq s$, and it holds $\alpha_{t+1}(x, R)<\infty$ for any $R>0$ by Theorem 2.1. Hence, letting $R_{n}=a^{M(n)} \rho\left|x_{n}-x\right|$, the preceding lemma, (ii) implies $\varlimsup_{\lim _{n}} \alpha_{l}\left(x_{n}, R_{n}\right)<\infty$ for $1 \leqq l \leqq s+1$. Since we may take the numbers $R$ and $R_{n}$ so that $\bar{U} \leqq \Delta\left(x_{n}, R_{n}\right)$ and $R_{n} \leqq R-\left|x_{n}-x\right|$, the estimate $\left(\mathrm{E}-1^{\prime}\right)$ implies that $\overline{\lim }_{n}\left\|D_{x_{n}}^{k}\right\|<\infty$ for $0 \leqq k \leqq s$. Therefore, we have $\varlimsup_{n}\left\|R_{x_{n}}^{s}\right\|<\infty$ by (2.6).

Now, let $\rho$ be any positive number. The theorem holds for $\rho^{\prime}=\alpha^{M} \rho$ if $M$ is sufficiently large. Since $\alpha_{s+1}(y, \rho|y-x|)=$ $\alpha_{s+1}^{0, M-1}(y, \rho|y-x|)+\alpha_{s+1}\left(y, a^{M} \rho|y-x|\right)$, it follows from the preceding lemma, (i) that $\varlimsup_{\lim _{n}} \alpha_{s+1}\left(x_{n}, \rho\left|x_{n}-x\right|\right)=\varlimsup_{n} \alpha_{s+1}\left(x_{n}, \rho^{\prime}\left|x_{n}-x\right|\right)$. Therefore, the theorem holds for any positive number $\rho$.

An application of our theorem is the following:

Corollary 2.11 (A. G. O'Farrell [4]). If the set $U$ contains a sector $\left\{z \in \Delta(x, r): \theta_{1}<\arg (z-x)<\theta_{2}\right\}$, then the functions in $A(U)$ are $t$-times continuously differentiable at $x$ along the half lines $\{z \in \Delta(x, r): \arg (z-x)=\theta\}\left(\theta_{1}<\theta<\theta_{2}\right)$ provided $D_{x}^{t}$ is bounded.

The proof is obvious, for $\alpha_{t}(y, \rho|y-x|)=0$ at any point on the half lines if $\rho<\tan \theta_{0}, \theta_{0}=\min \left\{\theta-\theta_{1}, \theta_{2}-\theta, \pi / 2\right\}$. 
Note. Here we remark on the rational function space $R(X)$. In this case, the corresponding estimates are valid for $R(X)$, where we replace the set $U$ by the set $X$. The proof is the same if we use the set $R(X)_{0}$ instead of the set $A(U ; x, y)$; where $R(X)_{0}$ is the set of the functions which are analytic on some neighborhood of $X$. Therefore, all analogy results of this section are valid for $R(X)$.

As already noted, the boundedness of $D_{x}^{t}$ does not guarantee the existence of a sequence $x_{n}$ with the property considered above. However, we have proved the following in [3] (J. L. Wang also proved this; cf. Theorem 2.13):

TheOREM 2.12. Let $x \in \bar{U}$ and suppose $\left\|D_{x}^{1}\right\|<\infty$. Denote by $m$ the 2-dimensional Lebesgue measure. Then there is a measurable set $E$ of $U$ such that

(i) $\lim _{r \rightarrow 0} \frac{m(E \cap \Delta(x, r))}{m(\Delta(x, r))}=1$,
(ii) $\lim _{\substack{y \rightarrow x \\ y \in E}} \frac{f(y)-f(x)}{y-x}=f^{\prime}(x)$ uniformly for $f \in A(U), \quad\|f\| \leqq 1$.

Roughly, this fact shows that the functions in $A(U)$ are differentiable at $x$ along almost all sequences provided $D_{x}^{1}$ is bounded. In high order case, the situation is quite different. We can construct an example such that $D_{x}^{t}$ are bounded for all $t \geqq 0$ but, for any sequence $x_{n}$ converging to $x$, the derivative $f^{\prime}\left(x_{n}\right)$ of a function $f$ in $A(U)$ is not continuous at $x$ (cf. $\S 3$, Example 3). For $R(X)$, A. G. O'Farrell ([5]) constructed a more extreme example such that $\left\|D_{x}^{1}\right\|=\infty$ for all $x \in X$ except only one point of $X$ at which $D_{x}^{t}$ is bounded for all $t \geqq 0$. In spite of this circumstance, J. L. Wang showed the following:

Theorem 2.13 (Wang [6]). For $u \in C$ and $f \in A(U)$, put $\left(\Delta_{u} f\right)(z)=f(z+u)-f(z)$ if $z, z+u \in \bar{U}$. And, inductively, put $\left(\Delta_{u}^{t} f(z)=\left(\Delta_{u}^{t-1} f\right)(z+u)-\left(\Delta_{u}^{t-1} f\right)(z)\right.$ if $z, z+u, \cdots, z+t u \in \bar{U}$. Let $x \in \bar{U}$ and suppose $\left\|D_{x}^{t}\right\|<\infty$. Denote by $m$ the 2-dimensional Lebesgue measure. There is a measurable set $F$ such that

(i) $x, x+u, \cdots, x+t u \in \bar{U}$ for $u \in F$,

(ii) $\lim _{r \rightarrow 0} \frac{m(F \cap \Delta(0, r))}{m(\Delta(0, r))}=1$,

(iii) $\lim _{\substack{u \rightarrow 0 \\ u \in F}} \frac{\left(\Delta_{u}^{s} f\right)(x)}{u^{t}}=f^{(s)}(x)$ uniformly for $f \in A(U),\|f\| \leqq 1$. 
REMARK. To be exact, Theorem 2.12 (resp., Theorem 2.13) has been proved only when the limit of (ii) (resp., (iii)) is weak-* limit in the paper [3] (resp., [6]). However, with a little more effort, one can prove the theorems in the above form.

The corresponding our result Theorem 2.13 is as follows;

Theorem 2.14. Let $x \in \bar{U}$ and suppose $\left\|D_{x}^{t}\right\|<\infty$. Let $u_{n} \in C \backslash\{0\}$ a sequence such that $u_{n} \rightarrow 0$ and $x+u_{n}, \cdots, x+t u_{n} \in \bar{U}$. Then,

$$
\frac{1}{t !} \lim _{n \rightarrow \infty} \frac{\left(\Delta_{u_{n}}^{t} f\right)(x)}{u_{n}^{t}}=D_{x}^{t} f
$$

for all $f \in A(U)$ (resp., uniformly for $f \in A(U),\|f\| \leqq 1$ ) if and only if

$$
\left.\varlimsup_{n \rightarrow \infty} \frac{\alpha_{1}\left(x+k u_{n}, \rho\left|u_{n}\right|\right)}{\left|u_{n}\right|^{t}}<\infty \quad \text { (resp., } \lim _{n \rightarrow \infty}=0\right)
$$

for all $k=1, \cdots, t$. Here, $\rho>0$ is any fixed number.

Proof. Since $\Delta_{u}^{t} f=t ! u^{t} D_{x}^{t} f+\Delta_{u}^{t}\left(R_{u}^{t} f\right)$ and

$$
\Delta_{u}^{t}\left(R_{x}^{t} f\right)=\sum_{k=1}^{t}(-1)^{k}\left(\begin{array}{l}
t \\
k
\end{array}\right)\left(R_{x}^{t} f\right)(x+k u),
$$

the "if" part is a simple consequence of the estimate $\left(\mathrm{E}-3^{\prime}\right)$. The "only if" part follows from the following estimate. The details are the same as before and will be omitted.

Estimate 2.15. Let $x \in \bar{U}$ and let $u$ be a nonzero complex number with $x+u, \cdots, x+t u \in \bar{U}$. Then, for $f \in A(U)$ and $0<\sigma<1$, it follows that

$$
\sup _{\|f\| \geqq 1}\left|\Delta_{u}^{t}\left(R_{x}^{t} f\right)(x)\right| \geqq \frac{C_{7} \alpha_{1}(x+l u, \sigma|u|)}{\alpha_{1}(x+l u, \sigma|u|)+C_{3}}
$$

for $l=1,2, \cdots, t$, where $C_{3}$ is the same constant as before and $C_{7}=C(a, \sigma, t)$ is an absolute constant uhich is positive if $\sigma>0$ is small.

The analogy results are valid for the space $H^{\circ}(U)$ of bounded analytic functions, but the proof is not similar. It follows from the results for $R(X)$. We shall mention it briefly.

First we define the analytic capacity, or $\gamma$-capacity; the definition is similar to $\alpha$-capacity, where we only replace the condition "continuous functions" by "Borel functions;" i.e., the analytic capacity, $\gamma(E)$, of a set $E$ in the plane is defined by 


$$
\gamma(E)=\sup \left\{\left|f^{\prime}(\infty)\right|: f \in \mathscr{A}(E)\right\},
$$

where $\mathscr{A}(E)$ is the set of all Borel functions $f$ on the sphere $S^{2}$ such that $f$ is analytic outside of a compact subset of $E$ and $\|f\|_{S^{2}} \leqq 1, f(\infty)=0$. The following properties are easily seen:

$$
\begin{gathered}
\alpha(E) \leqq \gamma(E), \text { and } \alpha(V)=\gamma(V) \text { if } V \text { is an open set. } \\
\gamma(K)=\inf \{\gamma(V): V \text { open, } V \supseteqq K\} \text { if } K \text { is a compact set . }
\end{gathered}
$$

If we replace the open annuli $E_{n}(x, r)$ by the closed annuli $\bar{E}_{n}(x, r)$, the notation $\gamma_{t}(x, r)_{U}$ is defined in the same way as $\alpha_{t}(x, r)_{U}$. However, the derivative $f^{(t)}(x)$ for $f \in H^{\infty}(U)$ is not defined so easily. This was defined by O'Farrell [4] using the device that T. W. Gamelin and J. Garnett found and used to define the distinguish homomorphisms $H^{\infty}(U)$. For our present purpose, we shall state it here: Let $H^{\infty}(U ; x)$ be the set of all Borel functions $f$ such that $f \in H^{\infty}(U)$ and $f$ is analytic in a neighborhood of $x$. Then it holds that

(2.9) (Gamelin and Garnett [7]) for each function $f \in H^{\infty}(U)$, there is a sequence $f_{n} \in H^{\infty}(U ; x)$ such that $\left\|f_{n}\right\|_{S^{2}} \leqq\|f\|_{U}$ and $f_{n}$ converges to $f$ pointwisely on $U$.

Now, for $f \in H^{\infty}(U ; x)$, we define $D_{x}^{t} f$ by $D_{x}^{t} f=(1 / t !) f^{(t)}(x)$. The lower estimate of the norm $\left\|D_{x}^{t}\right\|$ will be obtained in the same way. In particular, $\left\|D_{x}^{t}\right\|=\infty$ if $\gamma_{t+1}(x, r)=\infty$. Conversely, suppose $\gamma_{t+1}(x, r)<\infty$. Then, by (2.8), there is a compact set $X$ with the properties: $X \subseteq U \cup\{x\}, x \in X$ and $\gamma_{t+1}(x, r)_{X}<\infty$. It follows from (2.7) that $\alpha_{t+1}(x, r)_{X} \leqq \gamma_{t+1}(x, r)_{X}$. Therefore, $D_{x}^{t}$ is bounded on $R(X)$. Using Hahn-Banach's extension theorem and Riesz's representation theorem, there is a measure $\mu$ on $X$ such that $\int f d \mu=D_{x}^{t} f$ for $f \in R(X)$. If $\mu$ has the form $\mu=\nu+c \delta_{x} \quad\left(\delta_{x}\right.$ is the unit point measure at $x)$, we employ $v+c\left((z-x)^{t} / t\right.$ ! $) \mu$ as a new measure $\mu$ so that $\mu$ has no point mass at $x$. Now, we put $D_{x}^{t} f=\int f d \mu$ for $f \in H^{\infty}(U)$. We claim that $D_{x}^{t} f$ is determined independently of the choice of the measure $\mu$. Since $X \subseteq U \cup\{x\}$, we have $H^{\infty}(U ; x) \subseteq$ $R(X)$. Noting (2.9), dominate convergence theorem yields that

$$
D_{x}^{t} f=\lim _{n} \int f_{n} d \mu=\frac{1}{t !} \lim _{n} f_{n}^{(t)}(x)
$$

for any sequence $f \in H^{\infty}(U ; x)$ with $\left\|f_{n}\right\| \leqq\|f\|, f_{n} \rightarrow f$ on $U$. The expression shows that $D_{x}^{t} f$ depends neither on the measures $\mu$ nor on the sequence $\left\{f_{n}\right\}$. And we also obtained the following; 


$$
\left\|D_{x}^{t}\right\|_{H^{\infty}(U)} \leqq\left\|D_{x}^{t}\right\|_{R(X)}
$$

Since $\gamma(x, r)_{U}=\inf _{X} \gamma(x, r)_{X}$ by (2.8) (precisely, we also need (2.9)), and since $\alpha(x, r)_{X} \leqq \gamma(x, r)_{X}$, the upper estimate of $\left\|D_{x}^{t}\right\|_{H^{\infty}(U)}$ follows from the corresponding estimate of $\left\|D_{x}^{t}\right\|_{R(X)}$. One can obtain the other corresponding estimates for $H^{\infty}(U)$ in the same way. Hence, all analogy results of this section are valid also for $H^{\infty}(U)$.

3. Examples. We shall make some examples of $U$ concerning the space $A(U)$. As for the examples, the situations in the cases $R(X)$ and $H^{\infty}(U)$ are different. We shall also remark on it.

EXAMPLE 1. Let $U=\Delta(0,1) \backslash\left(\bigcup_{n=1}^{\infty} \Delta\left(i / 2^{n}, \varepsilon_{n}\right)\right)$. If $\varepsilon_{n} \leqq 2^{-(n+2)}$, then the $\operatorname{discs} U\left(i / 2^{n}, \varepsilon_{n}\right)$ are mutually disjoint and $0 \in b U$. So

$$
\begin{aligned}
\alpha_{t}\left(0, \frac{2}{3}, \frac{1}{2}\right) & =2^{t+1} \sum_{n=0}^{\infty}\left(\frac{2}{3} \cdot 2^{n}\right)^{t} \alpha\left(E_{n}\left(0, \frac{2}{3}, \frac{1}{2}\right) \backslash U\right) \\
& =2\left(\frac{4}{3}\right)^{t} \sum_{n=0}^{\infty} \varepsilon_{n+1} 2^{n t} .
\end{aligned}
$$

Thus, if $\varepsilon_{n}=2^{-(s+2) n}$, then $\left\|D_{0}^{s}\right\|<\infty$ and $\left\|D_{0}^{s+1}\right\|=\infty$; that is, all functions in $A(U)$ are of class $C^{s}$ on the interval $(-1,1)$ of the real axis, but there is a function in $A(U)$ which is not $s+1$ times differentiable at 0 . As another case, if $\varepsilon_{n}=2^{-\left(n^{2}+2\right)}$, then $\left\|D_{0}^{t}\right\|<\infty$ for all $t$; that is, all functions in $A(U)$ are of class $C^{\infty}$ on the interval $(-1,1)$.

EXAMPLE 2. Modifying the above example, it is possible to construct an example such that the interval lies entirely in the boundary of $U$ : To do this, we let

$$
U=\Delta(0,1) \backslash\left(\bigcup_{n=1}^{\infty} \bigcup_{k=-n}^{n} \Delta\left(\frac{k}{n}\left(1-\frac{1}{2^{n}}\right)+\frac{i}{2^{n}}, \varepsilon_{n}\right)\right)^{-}
$$

for $\varepsilon_{n} \leqq 2^{-(n+2)}$. Then the interval $J=(-1,1)$ on the real axis lies in $b U$. We claim that the functions in $A(U)$ are of class $C^{\infty}$ on $J$ if we set $\varepsilon_{n}=2^{-n^{2}} / 4 n$. Put

$$
w_{n k}=\frac{k}{n}\left(1-\frac{1}{2^{n}}\right)+\frac{i}{2^{n}} .
$$

We note that the sum of the arc length of $b \Delta\left(w_{n k}, \varepsilon_{n}\right)$ is finite, for $\sum_{n=1}^{\infty} 2 n \cdot 2 \pi \varepsilon_{n} \leqq \pi \sum_{n=1}^{\infty} 2^{-n^{2}}<\infty$. Thus, if a function $f$ is continuous on the Riemann sphere $S^{2}$ and analytic in $S^{2} \backslash\left(\bigcup_{n=1}^{\infty} \bigcup_{k=-n}^{n} \Delta\left(w_{n k}, \varepsilon_{n}\right)\right)^{-}$, then the Cauchy's integral formula yields ${ }^{1}$

\footnotetext{
${ }^{1}$ Note: $J$ is $\alpha$-negligible as noted below.
} 


$$
f(z)=\sum_{n=1}^{\infty} \sum_{k=-n}^{n} \frac{1}{2 \pi i} \int_{\left|\zeta-w_{n k}\right|=\varepsilon_{n}} \frac{f(\zeta)}{\zeta-z} d \zeta
$$

for $z \notin\left(\bigcup_{n=1}^{\infty} \bigcup_{k=-n}^{n} \Delta\left(w_{n k}, \varepsilon_{n}\right)\right)^{-}$. We first compute $\alpha\left(\Delta\left(x, 2^{-N}\right) \backslash U\right)$ for $x,-1<x<1$ : Let $f \in \mathscr{A} \mathscr{C}\left(\Delta\left(x, 2^{-N}\right) \backslash U\right)$. By the above formula, we have

$$
f^{\prime}(\infty)=\sum_{n, k} \frac{1}{2 \pi i} \int_{\left|\zeta-w_{n k}\right|=\zeta_{n}} f(\zeta) d \zeta
$$

By noting the analyticity of $f$, it suffice to sum up the integrals only for $n, k$ with $\Delta\left(x, 2^{-N}\right) \cap \Delta\left(w_{n k}, \varepsilon_{n}\right) \neq \varnothing$. So we have $\left|f^{\prime}(\infty)\right| \leqq$ $\sum_{n=N}^{\infty} 2 n \cdot \varepsilon_{n}$, and hence, $\alpha\left(\Delta\left(x, 2^{-N}\right) \backslash U\right) \leqq \sum_{n=N}^{\infty} 2 n \varepsilon_{n} \leqq \sum_{p=1}^{\infty} 2^{-N^{2}-p}=2^{-N^{2}}$. Now we compute $\alpha_{t}(x, r, 1 / 2) / r^{k}$ for small $r>0$. Let $M$ be the maximum integer with $r \leqq 2^{-M}$. Then $E_{n}(x, r, 1 / 2) \subset \Delta\left(x, 2^{-M-n}\right)$. Thus, noting $2^{-M-1}<r$, we have

$$
\begin{aligned}
\frac{\alpha_{t}(x, r, 1 / 2)}{r^{k}} & \leqq 2^{(M+1) k} \cdot 2^{t+1} \sum_{n=0}^{\infty} 2^{-(M+n)^{2}} \cdot 2^{(M+n+1) t} \\
& \leqq 2^{(M+1)(k+t)} \cdot 2^{t+1} \sum_{n=0}^{\infty} 2^{-M^{2}+n(t-2 M)}=2^{(M+1)(k+t)+t+1} \cdot \frac{2^{-M^{2}}}{1-2^{t-2 M}} .
\end{aligned}
$$

Therefore, if $r \rightarrow 0$, then $M \rightarrow \infty$ and we have

$$
\frac{\alpha_{t}(x, r, 1 / 2)}{r^{k}} \longrightarrow 0 ;
$$

the convergence is uniform on any compact subset of the interval $J=(-1,1)$. This shows that the functions in $A(U)$ are of class $C^{\infty}$ on the interval $J$.

In this example, the fact that $J$ is an interval in not essential. We have only needed the property of $J$ that every continuous function $f$ on $S^{2}$ which is analytic on an open set $V$ can be approximated uniformly by the continuous functions on $S^{2}$ which are analytic on some neighborhoods of $J \cup V$. The sets $J$ with this property are said to be $\alpha$-negligible. As is well-known, a finite number of piecewise twice continuously differentiable curve is $\alpha$-negligible. Therefore, starting from a compact $\alpha$-negligible set $J$, we can construct a similar example of $U$ in the following way; first, take an open set $D$ with $D \supseteqq J$, and take a sequence $\left\{z_{k}\right\}$ in $D \backslash J$ whose cluster points are in $J$; and then, choose a family of disjoint closed dises $\Delta_{k}$ in $D \backslash J$ with the centers $z_{k}$, and put $U=D \backslash\left(J \cup\left(\bigcup_{k} \Delta_{k}\right)\right)$. Then the smoothness on $J$ of the functions $f$ in $A(U)$ is obtained by accelerating the speed of the radii of $\Delta_{k}$ tending to zero. To construct a similar example for the case $R(X)$, we can take any compact set 
$J$ with no interior. For the case $H^{\infty}(U)$, we must take a $\gamma$-negligible set as $J . \quad$ A set $J$ is $\gamma$-negligible if there is a constant $M>0$ with the following property; for every bounded function $f$ on $S^{2}$ which is analytic on an open set $V$, there is a sequence of bounded functions $f_{n}$ such that $f_{n}$ are analytic on some neighborhood of $V \cup J$, $\left\|f_{n}\right\|_{\infty} \leqq M\|f\|_{\infty}$ and $f_{n}$ converges to $f$ pointwisely on $U$. Since every compact $\gamma$-negligible set is totally disconnected, we can not take any arc as the set $J$ in the case $H^{\infty}(U)$.

ExAmPLe 3. Here we give an example of $U$ such that $\left\|D_{0}^{t}\right\|<\infty$ at the origin $0 \in b U$ for all order $t$, but $\overline{\lim }_{n}\left\|D_{x_{n}}^{1}\right\|=\infty$ for any sequence $x_{n} \in \bar{U}$ converging to the origin 0 : Let $J$ be an open annulus $\{z: r<|z|<R\}$. It is known that, for any small $\varepsilon>0$ and large $\lambda>0$, there is a finite number of disjoint closed discs $\Delta_{k}$ such that $\Delta_{k} \subset J, \quad \alpha\left(\bigcup_{k} \Delta_{k}\right)<\varepsilon$ and $\alpha_{2}(x, r, \alpha)_{J}>\lambda$ for any $x \in \bar{J}$ (for instance, cf. [2; Example 1]). Now, for each $n$, deleting a finite number of closed disjoint discs $\Delta_{k}^{(n)}$ from $E_{n}(x, 1, a)$, we can make an open set $U=\Delta(0,1) \backslash\left(\bigcup_{n, k} \Delta_{k}^{(n)}\right)^{-}$such that $\alpha\left(E_{n}(0,1, a) \backslash U\right)<a^{n}$ and

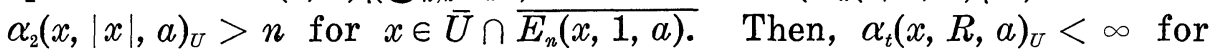
all $t$, and $\lim _{n} \alpha_{2}\left(x_{n},\left|x_{n}\right|, a\right)=\infty$ if $x_{n} \rightarrow 0$. Therefore, Theorem 2.2 shows that this is a desired example of $U$. Although the above constrction is somewhat rough, it is not difficult to make this precise.

4. Proof of the estimates (I). In this section we shall estimate the norms $\left\|D_{x}^{t}\right\|$ and $\left\|D_{y}^{s} R_{x}^{t}\right\|(t \geqq s)$ from below. To do this we use the following property of $\alpha$-capacity (cf. [1; Chap. VIII]): Let $K$ be a compact set in the complex plane $C$, and let $f$ be a continuous function on the Riemann sphere $S^{2}$ which is analytic in the outside of the set $K$ and vanishes at $\infty$. Then

$$
|f(z)| \leqq \frac{\alpha(K)}{d(z, K)}\|f\| \text { for } z \in S^{2} \backslash K ;
$$

where $d(z, K)$ denotes the distance from $z$ to $K$. Moreover, let $f(z)=A_{1} /(z-x)+A_{2} /(z-x)^{2}+A_{3} /(z-x)^{3}+\cdots$ be the Laurent expansion at $\infty \in S^{2}$ for $K \subset \Delta(x, r)$. It follows that

$$
\left|A_{k}\right| \leqq e k r^{k-1} \alpha(K)\|f\|
$$

(cf. [1; Chap. VIII]). Also, it follows that

$$
\left|A_{k}\right| \leqq r^{k}\|f\| \text {. }
$$

The latter formula is a consequence of an easy estimate of the integral $A_{k}=1 / 2 \pi i \int_{|z-x|=r}(z-x)^{k-1} f(z) d z$. 
Now, let $x \in \bar{U}$. We shall estimate the norm $\left\|D_{x}^{t}\right\|$. Let $r>0$ and $0<a<1$ be fixed, and put $X_{n}=E_{n}(x, r)$. For $\varepsilon>0$ and a positive integer $N$, take a function $f_{n} \in \mathscr{A} \mathscr{C}\left(X_{n} \backslash U\right)$ with

$$
\alpha\left(X_{n} \backslash U\right)-\left(r a^{n}\right)^{t+1} \frac{\varepsilon}{N+1} \leqq f^{\prime}(\infty) \leqq \alpha\left(X_{n} \backslash U\right)
$$

Let $f_{n}(z)=f_{n}^{\prime}(\infty) /(z-x)+A_{2} /(z-x)^{2}+A_{3} /(z-x)^{3}+\cdots$ be the Laurent expansion at $\infty$, and put

$$
\begin{aligned}
g_{n}(z)= & A_{t+1}+(z-x) A_{t}+\cdots+(z-x)^{t-1} A_{2} \\
& +(z-x)^{t} f_{n}^{\prime}(\infty)-(z-x)^{t+1} f_{n}(z) .
\end{aligned}
$$

We note that $g_{n}$ is vanish at $\infty$ and analytic wherever $f_{n}$ is analytic. Thus $g_{n} \in A(U)$. By the estimate (4.3) and by $f_{n}^{\prime}(\infty) \leqq \alpha\left(X_{n} \backslash U\right) \leqq r a^{n}$, we have

$$
\left\|g_{n}\right\|=\left\|g_{n}\right\|_{X_{n}} \leqq(t+2)\left(r a^{n}\right)^{t+1} .
$$

If $z \notin \bar{X}_{n-1} \cup \bar{X}_{n} \cup \bar{X}_{n+1}$, then $d\left(z, X_{n}\right) \geqq r a^{n+1}-r \alpha^{n+2}$. Hence, we have by the formula $(4.1)$

$$
\left|g_{n}(z)\right| \leqq \frac{\alpha\left(X_{n} \backslash U\right)}{(1-\alpha) r a^{n+1}}(t+2)\left(r a^{n}\right)^{t+1}
$$

Now we put

$$
g(z)=\sum_{n=0}^{N} \frac{g_{n}(z)}{\left(r a^{n}\right)^{t+1}} .
$$

Then $g \in A(U)$ and $g$ attains the maximum modulus on $\bigcup_{n=0}^{N} \bar{X}_{n}$. Let $z \in \bar{X}_{k}$. Using the estimate (2) for $n$ with $n \neq k-1, k, k+1$, and the estimate (1) for $n=k-1, k, k+1$, we have

$$
|g(z)| \leqq \frac{t+2}{(1-a) a} \sum_{n=0}^{N} \frac{\alpha\left(X_{n} \backslash U\right)}{r a^{n}}+3(t+2) .
$$

Therefore,

$$
\|g\| \leqq(t+2)\left(\alpha_{1}(x, r)+3\right) .
$$

On the other hand,

$$
D_{x}^{t} g=\sum_{n=0}^{N} \frac{f_{n}^{t}(\infty)}{\left(r \alpha^{n}\right)^{t+1}} \geqq \sum_{n=0}^{N} \frac{\alpha\left(X_{n} \backslash U\right)}{\left(r a^{n}\right)^{t+1}}-\varepsilon .
$$

Thus, by letting $\varepsilon \rightarrow 0$, it follows that

$$
\left\|D_{x}^{t}\right\| \geqq \frac{(1-a) a^{t}}{t+2} \frac{\alpha_{t+1}^{0, N}(x, r)}{\alpha_{1}^{0, N}(x, r)+3} .
$$


This proves the estimate $(\mathrm{E}-1)$.

Next we shall estimate the norm $\left\|D_{y}^{s} R_{x}^{t}\right\|(t \geqq s)$. Let $x, y \in \bar{U}$ and $0<r<d=|y-x|$, and put $Y_{n}=E_{n}(y, r)$. For $\varepsilon>0$ and a positive integer $N$, take a function $f_{n} \in \mathscr{A} \mathscr{C}\left(Y_{n} \backslash U\right)$ with

$$
\alpha\left(Y_{n} \backslash U\right)-\left(r a^{n}\right)^{s+1} \frac{\varepsilon}{N+1} \leqq f_{n}^{\prime}(\infty) \leqq \alpha\left(Y_{n} \backslash U\right) .
$$

As above, let $g_{n}(z)=f_{n}^{\prime}(\infty) /(z-y)+A_{2} /(z-y)^{2}+A_{3} /(z-y)^{3}+\cdots$ be the Laurent expansion at $\infty$, and put

$$
\begin{aligned}
g_{n}(z)= & A_{s+1}+(z-y) A_{s}+\cdots+(z-y)^{s-1} A_{2} \\
& +(z-y)^{s} f_{n}^{\prime}(\infty)-(z-y)^{s+1} f_{n}(z) .
\end{aligned}
$$

Then $g_{n} \in A(U)$. By (1) and (2), we have

$$
\begin{gathered}
\left\|g_{n}\right\| \leqq(s+2)\left(r a^{n}\right)^{s+1} \\
\left|g_{n}(z)\right| \leqq \frac{\alpha\left(Y_{n} \backslash U\right)}{(1-a) a^{n+1}}(s+2)\left(r a^{n}\right)^{s+1} \quad \text { for } \quad z \notin \bar{Y}_{n-1} \cup \bar{Y}_{n} \cup \bar{Y}_{n+1}
\end{gathered}
$$

Here we consider the Laurent expansion at $\infty$ once more; this time, making a new center of the point $x$, let $g_{n}(z)=B_{1} /(z-x)+$ $B_{2} /(z-x)^{2}+\cdots$ at $\infty$. Put

$$
h_{n}(z)=B_{t+1}+(z-x) B_{t}+\cdots+(z-x)^{t} B_{1}-(z-x)^{t+1} g_{n}(z) .
$$

Then $h_{n}$ is analytic wherever $g_{n}$ is analytic. Hence $h_{n} \in A(U)$ and $\left\|h_{n}\right\|=\left\|h_{n}\right\|_{Y_{n}}$. Since $\bigcup_{n=0}^{N} \bar{Y}_{n} \subset \Delta(x, d+r)$, the estimate (4.3) and (1) yields

$$
\left\|h_{n}\right\| \leqq(t+2)(d+r)^{t+1}\left\|g_{n}\right\| \leqq(t+2)(s+2)(d+r)^{t+1}\left(r a^{n}\right)^{s+1} .
$$

If $z \notin Y_{n-1} \cup Y_{n} \cup Y_{n+1}$, then the estimates (4.2) and (5) yields

$$
\begin{aligned}
\left|h_{n}(z)\right| \leqq & \left\{e(t+1)(d+r)^{t}+(d+r) \cdot e t(d+r)^{t-1}+\cdots+(d+r)^{t} \cdot e\right. \\
& \left.+\frac{(d+r)^{t+1}}{(1-a) r a^{n+1}}\right\} \alpha\left(Y_{n} \backslash U\right) \cdot(s+2)\left(r a^{n}\right)^{s+1} \\
= & \left\{\frac{e(t+1)(t+2)}{2}+\frac{d+r}{(1-a) r a^{n+1}}\right\}(d+r)^{t} \alpha\left(Y_{n} \backslash U\right) \\
& \times(s+2)\left(r a^{n}\right)^{s+1} .
\end{aligned}
$$

Now we put

$$
h(z)=\sum_{n=0}^{N} \frac{h_{n}(z)}{\left(r a^{n}\right)^{s+1}} .
$$

Then $h \in A(U)$ and $h$ attains the maximum modulus at a point in 
$\bigcup_{n=0}^{N} \bar{Y}_{n}$. Let $z \in \bar{Y}_{k}$. Applying the estimate (7) for $n$ with $n \neq k-1$, $k, k+1$ and the estimate (6) for $n=k-1, k, k+1$, we have

$$
\begin{aligned}
|h(z)| \leqq & \frac{e(t+1)(t+2)(s+2)(d+r)^{t}}{2} \sum_{n=0}^{N} \alpha\left(Y_{n} \backslash U\right) \\
& +\frac{(s+2)(d+r)^{t+1}}{(1-a) a} \sum_{n=0}^{N} \frac{\alpha\left(Y_{n} \backslash U\right)}{r a^{n}}+3(t+2)(s+2)(d+r)^{t+1} .
\end{aligned}
$$

Since $\sum_{n=0}^{N} \alpha\left(Y_{n} \backslash U\right) \leqq \sum_{n=0}^{N} r a^{n} \leqq r /(1-a)$, it follows that

$$
\|h\| \leqq(s+2)(d+r)^{t+1}\left(\alpha_{1}^{0, N}(y, r)+\frac{3 t^{2}+12 t+23}{2(1-a)}\right) .
$$

On the other hand, it holds $\left(R_{x}^{t} h\right)(z)=-(z-x)^{t+1} \sum_{n=0}^{N} g_{n}(z) /\left(r a^{n}\right)^{s+1}$, so Leibniz' formula yields

$$
D_{y}^{s}\left(R_{x}^{t} h\right)=-\frac{1}{s !} \sum_{k=0}^{s}\left(\begin{array}{l}
s \\
k
\end{array}\right) \frac{(t+1) !}{(t-k+1) !}(y-x)^{t-k+1} \cdot \sum_{n=0}^{N} \frac{g_{n}^{(s-k)}(y)}{\left(r a^{n}\right)^{s+1}} .
$$

We note that $g_{n}^{(k)}(y)=k ! A_{s-k+1}$ for $0 \leqq k<s$ and $g_{n}^{(s)}(y)=s ! f_{n}^{\prime}(\infty)$. By the estimate (4.2) for $A_{k}$, we have

$$
\begin{aligned}
\left|\sum_{n=0}^{N} \frac{g_{n}^{(s-k)}(y)}{\left(r a^{n}\right)^{s+1}}\right| & \leqq(s-k) ! \sum_{n=0}^{N} \frac{\left|A_{k+1}\right|}{\left(r a^{n}\right)^{s+1}} \\
& \leqq 3(k+1)(s-k) ! \sum_{n=0}^{N} \frac{\alpha\left(Y_{n} \backslash U\right)}{\left(r a^{n}\right)^{s-k+1}}
\end{aligned}
$$

for $0 \leqq k<s$, and

$$
\sum_{n=0}^{N} \frac{g_{n}^{(s)}(y)}{\left(r a^{n}\right)^{s+1}}=s ! \sum_{n=0}^{N} \frac{f_{n}^{\prime}(\infty)}{\left(r a^{n}\right)^{s+1}} \geqq s !\left(\sum_{n=0}^{N} \frac{\alpha\left(Y_{n} \backslash U\right)}{\left(r a^{n}\right)^{s+1}}-\varepsilon\right) .
$$

Thus we have

$$
\begin{aligned}
\left|D_{y}^{s}\left(R_{x}^{t} h\right)\right| \geqq & d^{t+1}\left(\sum_{n=0}^{N} \frac{\alpha\left(Y_{n} \backslash U\right)}{\left(r a^{n}\right)^{s+1}}-\varepsilon\right) \\
& -\sum_{k=1}^{s} 3(k+1)\left(\begin{array}{c}
t+1 \\
k
\end{array}\right) d^{t-k+1} \sum_{n=0}^{N} \frac{\alpha\left(Y_{n} \backslash U\right)}{\left(r a^{n}\right)^{s-k+1}} .
\end{aligned}
$$

Consequently, letting $\varepsilon \rightarrow 0$, we have the following estimate:

$$
\begin{aligned}
\left\|D_{y}^{s} R_{x}^{t}\right\| \geqq & \frac{\left(\frac{d}{d+r}\right)^{t+1}}{(s+2)\left(\alpha_{1}^{0, N}(y, r)+\frac{3 t^{2}+12 t+23}{2(1-a)}\right)}\left((1-a) a^{s+1} \alpha_{s+1}^{0, N}(y, r)\right. \\
& \left.-\sum_{k=1}^{s} 3(k+1)\left(\begin{array}{c}
t+1 \\
k
\end{array}\right) d^{-k}(1-a) a^{s-k+1} \alpha_{s-k+1}^{0, N}(y, r)\right) .
\end{aligned}
$$

Putting $r=\sigma|y-x|=\sigma d(0<\sigma<1)$, it follows that 
\| $D_{y}^{s} R_{x}^{t} \|$

$$
\begin{aligned}
\geqq & \frac{(1-a) \alpha^{s+1}}{(s+2)(1+\sigma)^{t+1}} \cdot \frac{1}{\left(\alpha_{1}^{0, N}(y, \sigma|y-x|)+\frac{3 t^{2}+12 t+23}{2(1-a)}\right)} \\
& \times\left(\alpha_{s+1}^{0, N}(y, \sigma|y-x|)-\sum_{k=1}^{s} \frac{3(k+1)}{a^{k}}\left(\begin{array}{c}
t+1 \\
k
\end{array}\right) \frac{\alpha_{s-k+1}^{0, N}(y, \sigma|y-x|)}{|y-x|^{k}}\right) .
\end{aligned}
$$

Now the estimate (E-2) follows if we put

$$
C_{2}=\frac{(1-a) a^{s+1}}{(s+2) 2^{t+1}}\left(\leqq \frac{(1-a) a^{s+1}}{(s+2)(1+\sigma)^{t+1}}\right), \quad C_{3}=\frac{3 t^{2}+12 t+23}{2(1-a)} .
$$

The proof of the estimate (E-4) is similar. We put $s=0$ in above. Then, the expressions (3) and (8) are as follows;

$$
\begin{gathered}
g_{n}(z)=f_{n}^{\prime}(\infty)-(z-y) f_{n}(z), \\
h(z)=\sum_{n=0}^{N} \frac{h_{n}(z)}{r a^{n}} .
\end{gathered}
$$

Also, we note that $\left(R_{x}^{t} h\right)(z)=-(z-x)^{t+1} \sum_{n=0}^{N} g_{n}(z) / r a^{n}$. Let $u$ be a nonzero complex number with $x+u, \cdots, x+t u \in \bar{U}$. For a certain $l$ with $1 \leqq l \leqq t$, we put $y=x+l u$. Since $d(x+k u, \Delta(y, r)) \geqq|u|-r$ for $k \neq l$, it follows from the formula (4.1) and (4) that

$$
\left|\left(R_{x}^{t} h\right)(x+k u)\right| \leqq|k u|^{t+1} \sum_{n=0}^{N} \frac{2 \cdot \alpha\left(Y_{n} \backslash U\right)}{|u|-r} \leqq \frac{2 \sigma|k u|^{t+1}}{1-\sigma} \sum_{n=0}^{N} \frac{\alpha\left(Y_{n} \backslash U\right)}{r a^{n}}
$$

for $k \neq l$; where $r=\sigma|u|, 0<\sigma<1$. And, it follows that

$$
\left|\left(R_{x}^{t} h\right)(y)\right|=\left|(l u)^{t+1} \sum_{n=1}^{N} \frac{f_{n}^{\prime}(\infty)}{r a^{n}}\right| \geqq|l u|^{t+1}\left(\sum_{n=0}^{N} \frac{\alpha\left(Y_{n} \backslash U\right)}{r a^{n}}-\varepsilon\right) .
$$

Since $\Delta_{u}^{t}\left(R_{x}^{t} h\right)(x)=\sum_{k=1}^{N}(-1)^{k}\left(\begin{array}{c}t \\ k\end{array}\right)\left(R_{x}^{t} h\right)(x+k u)$, we have

$$
\begin{aligned}
\left|\Delta_{u}^{t}\left(R_{x}^{t} h\right)(x)\right| \geqq & \left\{\left(\begin{array}{l}
t \\
l
\end{array}\right) l^{t+1}-\frac{2 \sigma}{1-\sigma} \sum_{k=1}^{t}\left(\begin{array}{l}
t \\
k
\end{array}\right) \mid k^{t+1}\right\}|u|^{t+1} \sum_{n=0}^{N} \frac{\alpha\left(Y_{n} \backslash U\right)}{r a^{n}} \\
& -\varepsilon\left(\begin{array}{l}
t \\
l
\end{array}\right)|l u|^{t+1} \cdot
\end{aligned}
$$

We note that

$$
\left(\begin{array}{l}
t \\
l
\end{array}\right) l^{t+1}-\frac{2 \sigma}{1-\sigma} \sum_{k=1}^{t}\left(\begin{array}{l}
t \\
k
\end{array}\right) k^{t+1} \geqq 1-\frac{\sigma}{1-\sigma}(2 t)^{t+1} .
$$

Since $r+d=\sigma|u|+|l u| \leqq(t+1)|u|$, it follows from (9) that

$$
\|h\| \leqq 2\{(t+1)|u|\}^{t+1}\left(\alpha_{1}^{0, N}(y, \sigma|u|)+C_{3}\right) .
$$


Thus, by letting $\varepsilon \rightarrow 0$ and $N \rightarrow \infty$, we have

$$
\sup _{\|f\| \geqq 1}\left|\Delta_{u}^{t}\left(R_{x}^{t} f\right)(x)\right| \geqq \frac{C_{7} \alpha_{1}(x+l u, \sigma|u|)}{\alpha_{1}(x+l u, \sigma|u|)+C_{3}},
$$

where

$$
C_{7}=\frac{\left(1-\frac{\sigma}{1-\sigma}(2 t)^{t+1}\right)(1-a) \alpha}{2(t+1)^{t+1}} .
$$

This constant $C_{7}$ is positive when $\sigma>0$ is small. This proves the estimate (E-4).

5. Proof of the estimates (II). In this section we shall estimate the norms $\left\|D_{x}^{t}\right\|$ and $\left\|D_{y}^{s} R_{x}^{t}\right\|(t \geqq s)$ from above. The tool is the following Melnikov's estimate (cf. [1; Chap. VIII]): If $K$ is a compact set and if $f$ is a continuous function on the closure of the open annulus $J=\{z: r<|z|<R\}$ such that $f$ is analytic in $J \backslash K$, then

$$
\left|\int_{b J} f(z) d z\right| \leqq \frac{2 \pi c_{0}}{1-(r / R)}\|f\|_{J} \alpha(J \cap K),
$$

where $c_{0}$ is a universal constant and $b J$ denotes the boundary of $J$. Now, let $x \in \bar{U}$, and fix a number $R$ with $\bar{U} \subset \Delta(x, R)$. We already mentioned about the dense subspace $A(U ; x)$ of $A(U)$ in $\S 2$. In the following, we assume that the functions $f$ in $A(U ; x)$ are extended to continuous functions on the Riemann sphere $S^{2}$ so that $f$ are analytic at $x$ and vanishes outside of $\Delta(x, R)$; moreover, we may assume that the sup-norm $\|f\|_{s^{2}}$ are sufficiently closed to $\|f\|_{U}$.

First we shall estimate of the norm $\left\|D_{x}^{t}\right\|$. Take a function $f$ in $A(U ; x)$, which is analytic in $\Delta(x, \varepsilon)$ for suitably small $\varepsilon>0$. It follows that

$$
D_{x}^{t} f=\frac{1}{t !} f^{(t)}(x)=\frac{1}{2 \pi i} \int_{|z-x|=\varepsilon} \frac{f(z)}{(z-x)^{t+1}} d z .
$$

Since we may assume $\varepsilon=R a^{N}$ for a larger $N$, we have

$$
\left|D_{x}^{t} f\right| \leqq \frac{1}{2 \pi} \sum_{n=0}^{N}\left|\int_{b E_{n}(x, R)} \frac{f(z)}{(z-x)^{t+1}} d z\right|+\frac{1}{2 \pi}\left|\int_{|z-x|=R} \frac{f(z)}{(z-x)^{t+1}} d z\right| .
$$

The last integral is zero by the assumption about $f$. Hence, the Melnikov's estimate (5.1) yields

$$
\begin{aligned}
\left|D_{x}^{t} f\right| & \leqq \sum_{n=0}^{N} \frac{c_{0}}{1-a} \cdot \frac{1}{\left(r a^{n+1}\right)^{t+1}}\|f\|_{S^{2}} \alpha\left(E_{n}(x, R) \backslash U\right) \\
& \leqq c_{0} \alpha_{t+1}(x, R)\|f\|_{S^{2}}
\end{aligned}
$$


Since the sup-norm $\|f\|_{S^{2}}$ is closed to $\|f\|_{U}$, we obtain the following estimate:

$$
\left\|D_{x}^{t}\right\| \leqq c_{0} \alpha_{t+1}(x, R) .
$$

Now we shall estimate the norm $\left\|D_{y}^{s} R_{x}^{t}\right\|$. Let $x, y \in \bar{U}$, and fix a number $R$ with $\bar{U} \subset \Delta(x, R)$. This time, we assume that the functions $f$ in $A(U ; x, y)$ are extended to continuous functions on the complex plane so that $f$ are anrlytic at $x, y$ and $R_{y}^{s} f$ vanishes outside of $\Delta(x, R)$; where $A(U ; x, y)$ is the dense subspace of $A(U)$ which was defined in $\S 2$. Let $f \in A(U ; x, y)$, then $f$ is analytic in $\Delta(x, \varepsilon)$ for a suitably small $\varepsilon>0$. First we make some preparatory computations: It is easy to see that

$$
\begin{aligned}
& D_{x}^{k}\left(R_{y}^{s} f\right)=D_{x}^{k} f \quad \text { if } \quad k>s, \\
& D_{x}^{s}\left(R_{y}^{s} f\right)=D_{x}^{s} f-D_{y}^{s} f .
\end{aligned}
$$

Therefore, it follows from (2.5) that

$$
\begin{aligned}
D_{y}^{s}\left(R_{x}^{t} f\right) & =D_{y}^{s} f-\sum_{k=s}^{t} \frac{k !}{s !(k-s) !}(y-x)^{k-s} D_{x}^{k} f \\
& =-\sum_{k=s}^{t}\left(\begin{array}{c}
k \\
s
\end{array}\right)(y-x)^{k-s} D_{s}^{k}\left(R_{y}^{s} f\right) \\
& =-\sum_{k=s}^{t}\left(\begin{array}{c}
k \\
s
\end{array}\right) \frac{(y-x)^{k-s}}{2 \pi i} \int_{|z-x|=\varepsilon} \frac{\left(R_{y}^{s} f\right)(z)}{(z-x)^{k+1}} d z \\
& =-\frac{1}{2 \pi i} \int_{|z-x|=\varepsilon} \frac{(y-x)^{k-s}}{(z-x)^{k+1}} \sum_{k=s}^{t}\left(\begin{array}{c}
k \\
s
\end{array}\right)\left(R_{y}^{s} f\right)(z) d z .
\end{aligned}
$$

Differentiating $s$ times the following equation as functions of $y$;

$$
\sum_{k=0}^{t} \frac{(y-x)^{k}}{(z-x)^{k+1}}=\frac{1}{z-y}-\frac{(y-x)^{t+1}}{(z-x)^{t+1}(z-y)},
$$

it holds that

$$
\begin{aligned}
\sum_{k=s}^{t} & \frac{k !}{(k-s) !} \frac{(y-x)^{k-s}}{(z-x)^{k+1}} \\
\quad= & \frac{s !}{(z-y)^{s+1}}-s ! \sum_{k=0}^{s}\left(\begin{array}{c}
t+1 \\
k
\end{array}\right) \frac{(y-x)^{t-k+1}}{(z-x)^{t+1}(z-y)^{s-k+1}} .
\end{aligned}
$$

Thus we have

$$
\begin{aligned}
D_{y}^{s}\left(R_{x}^{t} f\right) & \\
= & -\frac{1}{2 \pi i} \int_{|z-x|=\varepsilon}\left(\frac{1}{(z-y)^{s+1}}-\sum_{k=0}^{s}\left(\begin{array}{c}
t+1 \\
k
\end{array}\right) \frac{(y-x)^{t-k+1}}{(z-x)^{t+1}(z-y)^{s-k+1}}\right) \\
& \times\left(R_{y}^{s} f\right)(z) d z \\
= & (y-x)^{t-s} \sum_{k=0}^{s}\left(\begin{array}{c}
t+1 \\
k
\end{array}\right) \frac{(y-x)^{s-k+1}}{2 \pi i} \int_{|z-x|=\varepsilon} \frac{\left(R_{y}^{s} f\right)(z)}{(z-x)^{t+1}(z-y)^{s-k+1}} d z .
\end{aligned}
$$


For simplicity, we put $F(z)=\left(R_{y}^{s} f\right)(z)$ and $g(z)=F(z) /(z-y)^{l}$, where $l=s-k+1$. The function $F(z)$ has $s+1$ zero's at $y$ and $l \leqq s+1$, so we note that the function $g(z)$ is analytic wherever $F$ is analytic. Now our purpose is to estimate the following integral:

$$
\begin{aligned}
I_{l} & =\frac{(y-x)^{l}}{2 \pi i} \int_{|z-x|=\varepsilon} \frac{F(z)}{(z-x)^{t+1}(z-y)^{l}} d z \\
& =\frac{(y-x)^{l}}{2 \pi i} \int_{|z-x|=\varepsilon} \frac{g(z)}{(z-x)^{t+1}} d z .
\end{aligned}
$$

For positive numbers $\rho, \delta$, there is a continuously differentiable function $h$ such that $0 \leqq h \leqq 1, h(z)=1$ for $|z| \leqq \rho, h(z)=0$ for $|z|>\rho+\delta$ and $\|\partial h / \partial \bar{z}\| \leqq 1 / \delta$; for example, we define $h$ by $h(z)=1$ for $|z| \leqq \rho,=1-2((|z|-\rho) / \delta)^{2}$ for $\rho<|z| \leqq \rho+\delta / 2,=2((|z|-\rho-\delta) / \delta)^{2}$ for $\rho+\delta / 2<|z| \leqq \rho+\delta$, and $=0$ for $|z|>\rho+\delta$.

Let $0<a<1$ and $0<r<|y-x|$, and let $M$ be the maximum integer with $R a^{M+1} \geqq 2|y-x|$, i.e., $M=\left[\log _{a} 2|y-x| / R\right]-1$ (Gaussian symbol). For the sake of $M \geqq-1$, we assume $|y-x| \leqq R / 2$. Here, we prepare a system of continuously differentiable functions $h_{n}$ for $n=\infty$ and for $-1 \leqq n \leqq M$ such that $0 \leqq h_{n} \leqq 1$,

$$
h_{\infty}(z)=\left\{\begin{array}{lll}
1 & \text { on } & \Delta(y, a r) \\
0 & \text { off } & \Delta(y, r)
\end{array}, \quad\left\|\frac{\partial h_{\infty}}{\partial \bar{z}}\right\| \leqq \frac{1}{(1-a) r}\right.
$$

and

$$
h_{n}(z)=\left\{\begin{array}{ccc}
1 & \text { on } & \Delta\left(x, R a^{n+1}\right) \\
0 & \text { off } & \Delta\left(x, R a^{n}\right)
\end{array}, \quad\left\|\frac{\partial h_{n}}{\partial \bar{z}}\right\| \leqq \frac{1}{(1-a) R a^{n}}\right.
$$

Moreover, we define a system of functions $G_{n}$ for $n=\infty$ and for $-1 \leqq n \leqq M$ by

$$
\begin{aligned}
G_{n}(z) & =\frac{1}{\pi} \iint \frac{g(\zeta)-g(z)}{\zeta-z} \frac{\partial h_{n}}{\partial \bar{\zeta}} d \xi d \eta \\
& =g(z) h_{n}(z)+\frac{1}{\pi} \iint g(\zeta) \frac{\partial h_{n}}{\partial \bar{\zeta}} \frac{1}{\zeta-z} d \xi d \eta ;
\end{aligned}
$$

where $\zeta=\xi+i \eta$. It is known that $G_{n}$ has the following properties (cf. [1; Chap. II]): Denoting by $\bar{A}(x, r)$ the closure of $\Delta(x, r)$,

(a) $G_{n}$ is analytic wherever $g$ is analytic;

(b) $G_{n}$ is analytic outside of $\bar{\Delta}\left(x, R a^{n}\right)$ for $-1 \leqq n \leqq M$, and $G_{\infty}$ is analytic outside of $\bar{\Delta}(y, r)$;

(c) $G_{n}(\infty)=0$;

(d) $G_{n-1}-G_{n}$ is analytic on $\Delta\left(x, R a^{n+1}\right) \cup\left(S^{2} \backslash \bar{\Delta}\left(x, R a^{n}\right)\right)$ for $0 \leqq n \leqq M$, and $G_{M}-G_{\infty}$ is analytic on $\Delta(y, a r) \cup\left(S^{2} \backslash \bar{\Delta}\left(x, R a^{M}\right)\right)$. 
Moreover, there are several estimates made from the formula (3); we shall state them in order. For simplicity, we put $X_{n}=E_{n}(x, R)$ and $Y_{n}=E_{n}(y, r)$. Since $\partial h_{n} / \partial \bar{\zeta} \neq 0$ only on $X_{n}$, it follows that

$$
\begin{aligned}
\left|\left(G_{n-1}-G_{n}\right)(z)\right| & \leqq|g(z)| \cdot\left|1-h_{n}(z)\right|+\frac{1}{\pi}\|g\|_{X_{n-1} \cup X_{n}} \\
& \times\left\|\frac{\partial\left(h_{n-1}-h_{n}\right)}{\partial \bar{\zeta}}\right\| \iint_{\measuredangle\left(x, R a^{n-1}\right)} \frac{d \xi d \eta}{|\zeta-z|}, \\
\left|G_{n}(z)\right| \leqq|g(z)| & +\frac{1}{\pi}\|g\|_{X_{n}}\left\|\frac{\partial h_{n}}{\partial \bar{\zeta}}\right\| \iint_{\measuredangle\left\langle x, R a^{n}\right)} \frac{d \xi d \eta}{|\zeta-z|} .
\end{aligned}
$$

Here, $\quad\left\|\partial\left(h_{n-1}-h_{n}\right) / \partial \bar{\zeta}\right\|=\max \left(\left\|\partial h_{n-1} / \partial \bar{\zeta}\right\|,\left\|\partial h_{n} / \partial \bar{\zeta}\right\|\right) \leqq 1 /(1-a) R a^{n}$, and, for a measurable set $E$, it is easy to see that $\iint_{E} d \xi d \eta /|\zeta-z| \leqq$ $\iint_{\Delta(z, \delta)} d \xi d \eta /|\zeta-z|=2 \pi \delta$, where $\Delta(z, \delta)$ is the open disc whose area is the same as $E$ 's. Noting these facts and that $|y-x|<R a^{n+1} / 2$ for $n \leqq M$, we have

$$
\begin{gathered}
\left\|G_{n-1}-G_{n}\right\|_{X_{n}} \leqq\left(1+\frac{1}{\pi} \cdot \frac{1}{(1-a) R a^{n}} \cdot 2 \pi R a^{n-1}\right) \cdot \frac{\|F\|}{\left(R a^{n+1}-\frac{R a^{n+1}}{2}\right)^{l}}, \\
\left\|G_{n}\right\|_{X_{n}} \leqq\left(1+\frac{1}{\pi} \cdot \frac{1}{(1-a) R a^{n}} \cdot 2 \pi R a^{n}\right) \cdot \frac{\|F\|}{\left(R a^{n+1}-\frac{R a^{n+1}}{1}\right)^{l}} .
\end{gathered}
$$

Therefore, for $n \leqq M$, it follows that

$$
\begin{gathered}
\left\|G_{n-1}-G_{n}\right\|_{X n} \leqq \frac{3 \cdot 2^{l}}{(1-a) a} \frac{\|F\|}{\left(R a^{n+1}\right)^{l}}, \\
\left\|G_{n}\right\|_{X_{n}} \leqq \frac{3 \cdot 2^{l}}{1-a} \frac{\|F\|}{\left(R a^{n+1}\right)^{l}} .
\end{gathered}
$$

In the same way, have

$$
\begin{aligned}
\left\|G_{M}-G_{\infty}\right\| & =\left\|G_{M}-G_{\infty}\right\|_{\Delta\left(x, R a^{M}\right) \backslash \Delta(y, a r)} \\
& \leqq\left(1+\frac{1}{\pi} \cdot \frac{1}{(1-a) r} \cdot 2 \pi R a^{M}\right) \frac{\|F\|}{(r a)^{l}}, \\
\left\|G_{\infty}\right\|_{Y_{n}} & \leqq\left(1+\frac{1}{\pi} \cdot \frac{\|1\|}{(1-a) r} \cdot 2 \pi r\right) \frac{\|F\|}{\left(r a^{n+1}\right)^{l}} .
\end{aligned}
$$

Since $2|y-x|>R a^{M+2}$, it follows that

$$
\left\|G_{M}-G_{\infty}\right\| \leqq \frac{5|y-x|\|F\|}{(1-a) a(r a)^{l+1}},
$$




$$
\left\|G_{\infty}\right\|_{Y_{n}} \leqq \frac{3}{1-a} \cdot \frac{\|F\|}{\left(r a^{n+1}\right)^{l}} .
$$

Now, divide the integral (2) into the form

$$
\begin{aligned}
I_{l}= & \frac{(y-x)^{l}}{2 \pi i}\left\{\int_{|z-x|=\varepsilon} \frac{g-G_{-1}}{(z-x)^{t+1}} d z+\sum_{n=0}^{M} \int_{|z-x|=\varepsilon} \frac{G_{n-1}-G_{n}}{(z-x)^{t+1}} d z\right. \\
& \left.+\int_{|z-x|=\varepsilon} \frac{G_{M}-G_{\infty}}{(z-x)^{t+1}} d z+\int_{|z=x|=\varepsilon} \frac{G_{\infty}}{(z-x)^{t+1}} d z\right\} ;
\end{aligned}
$$

here there is not the second sum if $M=-1$. We estimate each integral separately

The first integral: Since $R_{y}^{s} f$ vanishes outside of $\Delta(x, R)$, it follows that $g\left(\partial h_{-1} / \partial \bar{z}\right) \equiv 0$. Thus, $G_{-1} \equiv g$. This shows that the first integral is zero.

The second integrals: Since $G_{n-1}-G_{n}$ is analytic in $\Delta\left(x, R a^{n+1}\right)$, the Cauchy's integral formula yields

$$
\begin{aligned}
\int_{|z-x|=\varepsilon} \frac{G_{n-1}-G_{n}}{(z-x)^{t+1}} d z & =\int_{|z-x|=R a^{n+1}} \frac{G_{n-1}-G_{n}}{(z-x)^{t+1}} d z \\
& =\int_{|z-x|=R a^{n+1}}-\int_{b X_{n-1}}-\int_{b X_{n}} .
\end{aligned}
$$

Since $\left(G_{n-1}-G_{n}\right) /(z-x)^{t+1}$ is analytic on $S^{2} \backslash \bar{\Delta}\left(x, R a^{n-1}\right)$ and its residue at $\infty$ is zero, the first integral is vanishing. Also, since $G_{n}$ is analytic on $X_{n-1}$, it follows that $\int_{b X_{n-1}}\left(G_{n-1}-G_{n}\right) /(z-x)^{t+1} d z=$ $\int_{b X_{n-1}}\left(G_{n-1} /(z-x)^{t+1}\right) d z$. Thus, by Melnikov's estimate (5.1) and the inequalities (4) and (5), we have

$$
\begin{aligned}
\left|\frac{1}{2 \pi i} \int_{|z-x|=\varepsilon} \frac{G_{n-1}-G_{n}}{(z-x)^{t+1}} d z\right| & \leqq \frac{3 \cdot 2^{l} c_{0}\|F\|}{(1-a)^{2}}\left(\frac{\alpha\left(X_{n-1} \mid U\right)}{\left(R a^{n}\right)^{t+1}\left(R a^{n}\right)^{l}}\right. \\
& \left.+\frac{1}{a} \cdot \frac{\alpha\left(X_{n} \backslash U\right)}{\left(R a^{n+1}\right)^{t+1}\left(R a^{n+1}\right)^{l}}\right)
\end{aligned}
$$

here there is not the first term in the right hand when $n=0$. Therefore,

$$
\begin{aligned}
& \left|\frac{1}{2 \pi i} \sum_{n=0}^{M} \int_{|z-x|=\varepsilon} \frac{G_{n-1}-G_{n}}{(z-x)^{t+1}} d z\right| \\
& \quad \leqq \frac{3 \cdot 2^{l} c_{0}\|F\|}{(1-a)^{2}}\left(\sum_{n=1}^{M} \frac{\alpha\left(X_{n-1} \backslash U\right)}{\left(R a^{n}\right)^{t+l+1}}+\frac{1}{a} \sum_{n=0}^{M} \frac{\alpha\left(X_{n} \backslash U\right)}{\left(R a^{n+1}\right)^{t+l+1}}\right),
\end{aligned}
$$

since $R a^{M+1} \geqq 2|y-x|>2 r$,

$$
\leqq \frac{3 \cdot 2^{l} c_{0}\|F\|}{(1-a)^{2}}\left(\frac{1}{a} \cdot \frac{\alpha\left(X_{M} \backslash U\right)}{\left(R a^{M+1}\right)^{t+l+1}}+\frac{2}{a} \sum_{n=0}^{M-1} \frac{\alpha\left(X_{n} \backslash U\right)}{\left(R a^{n+1}\right)^{t+l+1}}\right) .
$$


The third integral: Since we may assume $\varepsilon=R a^{N}$ for a large integer $N$, we have

$$
\int_{|z-x|=\varepsilon} \frac{G_{M}-G_{\infty}}{(z-x)^{t+1}} d z=\int_{|z-x|=R a M}-\sum_{n=M}^{N} \int_{b X_{n}} .
$$

The function $\left(G_{M}-G_{\infty}\right) /(z-x)^{t+1}$ is analytic on $S^{2} \backslash \bar{A}\left(x, R a^{M}\right)$ and its residue at $\infty$ is zero, so the first integral is vanishing. Also, since $G_{\infty}$ is analytic on $X_{M}$, it follows that

$$
\int_{b X_{M}} \frac{G_{M}-G_{\infty}}{(z-x)^{t+1}} d z=\int_{b X_{M}} \frac{G_{M}}{(z-x)^{t+1}} d z
$$

Therefore, Melnikov's estimate (5.1) and the inequalities (5) and (6) yield

$$
\begin{aligned}
& \left|\frac{1}{2 \pi i} \int_{|z-x|=\varepsilon} \frac{G_{M}-G_{\infty}}{(z-x)^{t+1}} d z\right| \\
& \quad \leqq \frac{3 \cdot 2^{l} c_{0}|| F||}{(1-a)^{2}} \cdot \frac{\alpha\left(X_{M} \mid U\right)}{\left(R a^{M+1}\right)^{l}\left(R a^{M+1}\right)^{t+1}}+\frac{5|y-x| c_{0}\|F\|}{(1-a)^{2} a(r a)^{l+1}} \sum_{n=M+1}^{N} \frac{\alpha\left(X_{n} \mid U\right)}{\left(R a^{n+1}\right)^{t+1}} .
\end{aligned}
$$

Here we may put $N=\infty$.

The fourth integral: Since the function $G_{\infty} /(z-x)^{t+1}$ is analytic on $S^{2} \backslash \bar{\Delta}(y, r)$ and its residue at $\infty$ is zero, the Cauchy's integral formula yields

$$
\begin{aligned}
\int_{|z-x|=\varepsilon} \frac{G_{\infty}}{(z-x)^{t+1}} d z & =\int_{|z-x|=r} \frac{G_{\infty}}{(z-x)^{t+1}} d z \\
& =\sum_{n=0}^{\infty} \int_{b Y_{n}} \frac{G_{\infty}}{(z-x)^{t+1}} d z ;
\end{aligned}
$$

in the last summation, we note that integrals on $b Y_{n}$ are vanishing for large integers $n$ because $G_{\infty}$ is analytic in a neighborhood of $y$. Thus, Melnikov's estimate (5.1) and the inequality (7) yield

$$
\begin{aligned}
& \left|\frac{1}{2 \pi i} \int_{|z-x|=\varepsilon} \frac{G_{\infty}}{(z-x)^{t+1}} d z\right| \leqq \frac{3 c_{0}\|F\|}{(1-a)^{2}} \sum_{n=0}^{\infty} \frac{\alpha\left(Y_{n} \backslash U\right)}{\left(r a^{n+1}\right)^{l}(|y-x|-r)^{t+1}} \\
& \quad \leqq \frac{3 c_{0}|| F \|}{(1-a)^{2}(|y-x|-r)^{t+1}} \sum_{n=0}^{\infty} \frac{\alpha\left(Y_{n} \backslash U\right)}{\left(r a^{n+1}\right)^{l}} .
\end{aligned}
$$

Put these estimates (8), (9) and (10) together, we have the following estimate: If $|y-x| \leqq R / 2$, then

$$
\begin{aligned}
\left|I_{l}\right| \leqq & c_{0}|y-x|^{l}|| R_{y}^{s} f \|\left(\frac{6 \cdot 2^{l}}{(1-a)^{2} \alpha} \sum_{n=0}^{M} \frac{\alpha\left(X_{n} \backslash U\right)}{\left(R a^{n+1}\right)^{t+l+1}}\right. \\
& +\frac{5|y-x|}{(1-a)^{2} a(r a)^{l+1}} \sum_{n=M+1}^{\infty} \frac{\alpha\left(X_{n} \backslash U\right)}{\left(R a^{n+1}\right)^{t+1}} \\
& \left.+\frac{3}{(1-a)^{2}(|y-x|-r)^{t+1}} \sum_{n=0}^{\infty} \frac{\alpha\left(Y_{n} \backslash U\right)}{\left(r a^{n+1}\right)^{l}}\right)
\end{aligned}
$$


where $M=\left[\log _{a} 2|y-x| / R\right]-1$ (Gaussian symbol) and $1 \leqq l \leqq s+1$.

Form this estimate, we obtain the following version: Let $r=\sigma|y-x|, 0<\sigma<1$. If $|y-x| \leqq R / 4^{l}$, then it holds

$$
\begin{aligned}
\left|I_{l}\right| \leqq & c_{0}\left\|R_{y}^{s} f\right\|\left(\frac{6 \cdot 2^{l}}{(1-a) a} \sqrt{\frac{|y-x|}{R}} \alpha_{t+1}(x, R)\right. \\
& +\left(\frac{1}{\sigma}\right)^{l+1} \frac{6}{(1-a) a^{l+2}} \alpha_{t+1}^{L, \infty}(x, R) \\
& \left.+\left(\frac{1}{1-\sigma}\right)^{t+1} \frac{3}{1-a} \frac{\alpha_{l}(y, \sigma|y-x|)}{|y-x|^{t-l+1}}\right),
\end{aligned}
$$

where $L=\left[(1-(1 / 2 l)) \log _{a}|y-x| / R\right]$ (Gaussian symbol).

Proof. For simplicity, we put $d=|y-x|$. Then $L$ is the maximum integer with $R(d / R)^{1-(1 / 2 l)} \leqq R a^{L}$. From the condition $|y-x| \leqq R / 4^{l}$, it follows that $2 d \leqq R(d / R)^{1-(1 / 2 l)} \leqq R a^{L}$. Hence, $0 \leqq L \leqq M+1$. Now, we transform the first term of (5.3) in the following way: Since $R(d / R)^{1-(1 / 2 l)} \leqq R a^{n+1}$ for $0 \leqq n<L$,

$$
\begin{aligned}
\frac{6 \cdot 2^{l}}{(1-a)^{2} a} \sum_{n=0}^{L-1} \frac{\alpha\left(X_{n} \backslash U\right)}{\left(R a^{n+1}\right)^{t+l+1}} & \leqq \frac{6 \cdot 2^{l}}{(1-a)^{2} a} \cdot \frac{1}{R^{l}\left(\frac{d}{R}\right)^{l-(1 / 2)}} \cdot \frac{1}{a^{t+1}} \sum_{n=0}^{L-1} \frac{\alpha\left(X_{n} \backslash U\right)}{\left(R a^{n}\right)^{t+1}} \\
& \leqq \frac{1}{d^{l}} \frac{6 \cdot 2^{l}}{(1-a) a} \sqrt{\frac{d}{R}} \alpha_{t+1}(x, R)
\end{aligned}
$$

and, since $2 d \leqq R a^{n+1}$ for $n \leqq M$,

$$
\begin{aligned}
\frac{6 \cdot 2^{l}}{(1-a)^{2} a} \sum_{n=L}^{M} \frac{\alpha\left(X_{n} / U\right)}{\left(R a^{n+1}\right)^{t+l+1}} & \leqq \frac{6 \cdot 2^{l}}{(1-a)^{2} a} \cdot \frac{1}{(2 d)^{l}} \cdot \frac{1}{a^{t+1}} \sum_{n=L}^{M} \frac{\alpha\left(X_{n} \backslash U\right)}{\left(R a^{n}\right)^{t+1}} . \\
& \leqq \frac{1}{d^{l}} \cdot \frac{1}{(1-a) \alpha} \alpha_{t+1}^{L, M}(x, R) .
\end{aligned}
$$

The second term of (5.3) is transformed in the following way:

$$
\frac{5 d}{(1-a)^{2} a(r a)^{l+1}} \sum_{n=M+1}^{\infty} \frac{\alpha\left(X_{n} \backslash U\right)}{\left(R a^{n+1}\right)^{t+1}} \leqq \frac{1}{d^{l}} \cdot\left(\frac{1}{\sigma}\right)^{l+1} \cdot \frac{5}{(1-a) a^{l+2}} \alpha_{t+1}^{M+1, \infty}(x, R) .
$$

And, the last term of (5.3) is transformed in the following way:

$$
\frac{3}{(1-\alpha)^{2}(d-r)^{t+1}} \sum_{n=0}^{\infty} \frac{\alpha\left(Y_{n} \backslash U\right)}{\left(r a^{n+1}\right)^{l}} \leqq \frac{1}{d^{l}} \cdot\left(\frac{1}{1-\sigma}\right)^{t+1} \cdot \frac{1}{1-a} \cdot \frac{\alpha_{l}(y, \sigma d)}{d^{t-l+1}}
$$

Hence, we obtain the estimate (5.4).

Now we turn back to the formula (1) and obtain the following estimate of the norm $\left\|D_{y}^{s} R_{x}^{t}\right\|:$ If $s \leqq t, 0<\sigma<1$ and $|y-x| \leqq$ $R / 4^{s+1}$, then it holds 


$$
\begin{aligned}
\left\|D_{y}^{s} R_{x}^{t}\right\| \leqq & c_{0}|y-x|^{t-s}\left\|R_{y}^{s}\right\|\left(\sqrt{\frac{|y-x|}{R}} \sum_{l=1}^{s+1}\left(\begin{array}{c}
t+1 \\
s-l+1
\end{array}\right) \frac{6 \cdot 2^{l}}{(1-a) a} \alpha_{t+1}(x, R)\right. \\
& +\sum_{l=1}^{s+1}\left(\begin{array}{c}
t+1 \\
s-l+1
\end{array}\right)\left(\frac{1}{\sigma}\right)^{l+1} \frac{6}{(1-a) a^{l+2}} \alpha_{t+1}^{L, \infty}(x, R) \\
& \left.+\left(\frac{1}{1-\sigma}\right)^{t+1} \frac{3}{1-a} \sum_{l=0}^{s+1}\left(\begin{array}{c}
t+1 \\
s-l+1
\end{array}\right) \frac{\alpha_{l}(y, \sigma|y-x|)}{|y-x|^{t-l+1}}\right) .
\end{aligned}
$$

The estimate $\left(\mathrm{E}-2^{\prime}\right)$ follows if we put

$$
C_{3}=\sum_{l=1}^{s+1}\left(\begin{array}{c}
t+1 \\
s-l+1
\end{array}\right) \frac{6 \cdot 2^{l} c_{0}}{(1-a) a}, \quad C_{4}=\sum_{l=1}^{s+1}\left(\begin{array}{c}
t+1 \\
s-l+1
\end{array}\right)\left(\frac{1}{\sigma}\right)^{l+1} \frac{6 c_{0}}{(1-a) a^{l+2}},
$$

and

$$
C_{5}=\frac{3 c_{0}}{1-a}\left(\frac{1}{1-\sigma}\right)^{t+1}
$$

Note. There is another way to estimate the norm $\left\|D_{y}^{s} R_{x}^{t}\right\|$ from above. We note it in short. By Leibniz's formula, it follows that

$$
\begin{aligned}
D_{y}^{s}\left(R_{x}^{t} f\right) & =D_{y}^{s}\left((z-x)^{t+1} \frac{R_{x}^{t} f}{(z-x)^{t+1}}\right) \\
& =\frac{1}{s !} \sum_{k=0}^{s}\left(\begin{array}{c}
s \\
k
\end{array}\right) \frac{(t+1) !}{(t-k+1) !}(z-x)^{t-k+1}(s-k) ! D_{y}^{s-k}\left(\frac{R_{x}^{t} f}{(z-x)^{t+1}}\right) \\
& =(z-x)^{t-s} \sum_{k=0}^{s}\left(\begin{array}{c}
t+1 \\
k
\end{array}\right)(z-x)^{s-k+1} D_{y}^{s-k}\left(\frac{R_{x}^{t} f}{(z-x)^{t+1}}\right)
\end{aligned}
$$

for $f \in A(U ; x, y)$. We may assume that $f$ is analytic in $\Delta(y, \varepsilon)$ for a small $\varepsilon>0$. Then, we have

$$
\begin{aligned}
& (z-x)^{s-k+1} D_{y}^{s-k}\left(\frac{R_{x}^{t} f}{(z-x)^{t+1}}\right) \\
& \quad=\frac{(z-x)^{s-k+1}}{2 \pi i} \int_{|z-y|=\varepsilon} \frac{R_{x}^{t} f}{(z-y)^{s-k+1}(z-x)^{t+1}} d z .
\end{aligned}
$$

Now, the way to estimate this integral is similar to that we have just seen. In this case, we put $g(z)=\left(R_{x}^{t} f\right)(z) /(z-x)^{t+1}$ and change the definition of $h_{\infty}$ so that $h_{\infty}$ has its support in a neighborhood of $x$. The estimate obtained by this is similar to that we have given in above except that the condition $0<\sigma<1$ is replaced by the condition $\sigma>1$.

NoTE. In his paper [8], O'Farrel investigated the bihavior of $t$-th order derivative $D_{y}^{t}$ at a point $x$ under the condition 
$\alpha_{t+\kappa+1}(x, r)<\infty$, where $0<\kappa<1$. A version of our estimate (5.5) is also applied to this case. The corresponding theorem is as follows:

TheOREM. Let $E$ be a subset of $\bar{U}$ and let $0<\kappa<1$. Suppose $\alpha_{t+\kappa+1}(x, r)<\infty$ for some $r>0$. Then, there is a constant $M>0$ such that

$$
\left\|D_{y}^{t}-D_{x}^{t}\right\| \leqq M|y-x|^{\kappa} \quad \text { for all } y \in E
$$

if and only if

$$
\sup _{y \in E} \frac{\alpha_{t+1}(y, \rho|y-x|)}{|y-x|^{\kappa}}<\infty .
$$

Here, $\rho>0$ is any fixed number.

To obtain the upper estimate, we put $L=\left[(1-(\beta / l)) \log _{a}|y-x| / R\right]$ in the proof of (5.4), where $1>\beta>\kappa>0$. A similar computations yield the following estimate: If $|y-x|<R / 2^{(s+1) / \beta}$, then it holds

$$
\begin{aligned}
\left\|D_{y}^{s} R_{x}^{t}\right\| \leqq & |y-x|^{t-s}|| R_{y}^{s}||\left(\frac{|y-x|^{\beta}}{R} C_{4} \alpha_{t+1}(x, R)\right. \\
& +|y-x|^{\kappa} 2^{(s+1) \kappa} C_{4} \alpha_{t+\kappa+1}^{L, \infty}(x, R) \\
& \left.+|y-x|^{\kappa} C_{5} \sum_{l=0}^{s+1}\left(\begin{array}{c}
t+1 \\
s-l+1
\end{array}\right) \frac{\alpha_{l}(y, \sigma|y-x|)}{|y-x|^{t-l+\kappa+1}}\right),
\end{aligned}
$$

where the constant $C_{3}, C_{4}$ and $C_{5}$ are the same as before. As for the lower estimate, we can use $(E-3)$.

6. Appendix. We already proved some properties of $\alpha_{t}(x, r, a)$ in $\S 2$. The proof, however, depends on the estimates of $\left\|D_{x}^{t}\right\|$ and $\left\|D_{y}^{s} R_{x}^{t}\right\|$. The purpose of this section is to indicate a direct proof of the following properties:

(i) If $\alpha_{t}(x, r, a)<\infty$ for some $r>0, \quad 0<a<1$, then $\alpha_{t}(x, r, a)<\infty$ for any $r>0,0<a<1$.

Suppose $\alpha_{t}(x, R)<\infty$ below ((ii) $\left.\sim(\mathrm{v})\right)$ :

(ii) $\lim _{r \rightarrow 0} \frac{\alpha(\Delta(x, r) \backslash U)}{r^{t}}=0$.

(iii) $\lim _{r \rightarrow 0} \frac{\alpha_{s}(x, r)}{r^{k}}=0$ for $s, k \geqq 0$ with $s+k \leqq t$.

In addition, let $0 \leqq s \leqq t$ and let $x_{n} \in \bar{U}, x_{n} \rightarrow x$ :

(iv) If $\varlimsup_{n \rightarrow \infty} \frac{\alpha_{s}\left(x_{n}, \rho\left|x_{n}-x\right|\right)}{\left|x_{n}-x\right|^{k}}<\infty$ (resp., $\lim _{n \rightarrow \infty}=0$ ) for some $\rho>0$, then the same holds for any $\rho>0$. 
(v) If $\varlimsup_{n \rightarrow \infty} \alpha_{s}\left(x_{n}, \rho\left|x_{n}-x\right|\right)<\infty$ for some $\rho>0$, then $\lim \alpha_{s}\left(x_{n}, R\right)<\infty$ for any $R>0$.

These properties are valid not only when the numbers $t, s$ and $k$ are integers but also when these are real numbers. The properties follow immediately from the following estimates:

$$
C_{8}^{-1} \alpha_{t}(x, r, b) \leqq \alpha_{t}(x, r, a) \leqq C_{8} \alpha_{t}(x, r, b),
$$

where $0<a, b<1$ and

$$
C_{8}=c_{0} \max \left\{\frac{\log _{a} b+2}{(1-b) b^{t}}, \frac{\log _{b} a+2}{(1-a) a^{t}}\right\} .
$$

$$
\begin{aligned}
& \alpha_{t}(x, r) \leqq \frac{2 c_{0}}{(1-a) a^{t}} \alpha_{t}^{k, \infty}(x, R) \quad \text { if } \quad r \leqq R a^{k} \\
& \frac{\alpha(\Delta(x, r) \backslash U)}{r^{t}} \leqq c_{0} \alpha_{t}(x, r) . \\
& \alpha_{t}(y, \rho|y-x|) \leqq \alpha_{t}\left(y, a^{N} \rho|y-x|\right) \\
& +\frac{N}{(1-a) a^{t}} \frac{\alpha(\Delta(x,(1+\rho)|y-x|) \backslash U)}{\rho^{t}|y-x|^{t} a^{N t}} . \\
& \alpha_{t}(y, r) \leqq \frac{2 c_{0}}{(1-a) a^{t}} \alpha_{t}(y, \rho|y-x|)+C_{10} \alpha_{t}(x, R),
\end{aligned}
$$

where

$$
\rho>\frac{2}{a-a^{2}}, R \geqq r+\rho|y-x| \text { and } C_{10}=\frac{3 c_{0}}{(1-a) a^{2 t}} .
$$

In fact, (i) is clear by (6.1). (ii) follows from (6.2) and (6.3), and (iii) follows from (6.2) and (2.3). By (6.2), it holds $\alpha_{s}\left(y, a^{N} \rho|y-x|\right) \leqq$ $\left(2 c_{0} /(1-a) a^{t}\right) \alpha_{t}(y, \sigma|y-x|)$ if $a^{N} \rho \leqq \sigma$. So (iv) follows from (6.4) and (ii). (v) is clear by (iv) and (6.5).

The estimate (6.4) has been proved in the proof of Lemma 2.10, (i). To prove the other estimates, we need the following inequality which is an easy application of the Melnikov's estimate (5.1) (cf. [1; Chap. VIII, Cor. 12.8]): If $E$ is a subset of the complex plane, then

$$
\alpha(\Delta(x, r) \backslash E) \leqq c_{0} \alpha\left(\Delta\left(x, r a^{M}\right) \backslash E\right)+\frac{c_{0}}{1-a} \sum_{n=0}^{M-1} \alpha\left(E_{n}(x, r, \alpha) \backslash E\right) .
$$

Now, the proof of (6.3) is at hand; letting $M \rightarrow \infty$ in (6.6), we have $\alpha(\Delta(x, r) \backslash E) \leqq c_{0} /(1-a) \alpha_{0}(x, r)$. So (2.3) yields (6.3). Since each $E_{k}(x, r, a)$ can be covered by two succesive $E_{m}(x, R, a)$ and $E_{m+1}(x, R, a)$, noting 


$$
\alpha\left(E_{k}(x, r, a) \backslash U\right) \leqq c_{0} /(1-a)\left(\alpha\left(E_{m}(x, R, a) \backslash U\right)+\alpha\left(E_{m+1}(x, R, a) \backslash U\right)\right)
$$

and $r a^{k} \geqq R a^{m+1}$, we have (6.2). Now we prove (6.5): For simplicity, we put $X_{m}=E_{m}(x, R, a)$ and $Y_{n}=E_{n}(y, r, a)$. Let $k$ be the minimum integer with $\rho|y-x| \geqq r a^{k}$. By (6.2), we have $\alpha_{t}^{k, \infty}(y, r) \leqq$ $2 c_{0} /\left((1-a) a^{t}\right) \alpha_{t}(y, \rho|y-x|)$. Now, for $0 \leqq n \leqq k$, it holds $r a^{n+1}>$ $|y-x|$ since $r a^{n}>\rho|y-x|$. So, each $Y_{n}$ can be covered by a finite number of $X_{m}$, say, $X_{p}, \cdots, X_{p+q}$. Since $Y_{n}$ intersects with $X_{p}$ and $X_{p+q}$, it holds $R a^{p+1}<r a^{n}+|y-x|$ and $R a^{p+q}>r a^{n+1}-|y-x|$. By $r a^{n} \geqq \rho|y-x|$, we have

$$
a^{q-1}>\frac{r a^{n+1}-|y-x|}{r a^{n}+|y-x|}>a^{2},
$$

and hence, $q \leqq 2$. Thus, noting $R a^{p+q+1} \leqq r a^{n+1}-|y-x|<r a^{n+1}$, we have

$$
\frac{\alpha\left(Y_{n} \backslash U\right)}{\left(r a^{n}\right)^{t}} \leqq \frac{c_{0}}{(1-a) a^{2 t}} \sum_{m=p}^{p+q} \frac{\alpha\left(X_{m} \backslash U\right)}{\left(R a^{m}\right)^{t}} .
$$

On the other hand, if $X_{n}$ intersects four of $Y_{n}$, say, $Y_{n-3}, \cdots, Y_{n}$, then it holds $R a^{m}>r a^{n-2}-|y-x|$ and $R a^{m+1}<r a^{n}+|y-x|$; that is,

$$
a<\frac{r a^{n}+|y-x|}{r a^{n-2}-|y-x|}, \quad \text { or } \quad r a^{n}<\frac{a+a^{2}}{1-a}|y-x|<\rho|y-x| .
$$

Hence, each $X_{m}$ does not intersect more than three of $Y_{n}, n \leqq N$. Thus, summing up the inequality, we have

$$
\alpha_{t}^{0, k-1}(y, r) \leqq \frac{3 c_{0}}{(1-a) \alpha^{2 t}} \alpha_{t}(x, R)
$$

This proves (6.5). The idea of the proof of (6.1) is the same as (6.5) and we shall omit its proof.

Note. Some results in this section would be well-known.

\section{REFERENCES}

1. T. W. Gamelin, Uniform Algebras. Prentice-Hall, New York, 1969.

2. A. P. Hallstrom, On bounded point derivations and analytic capacity, J. Functional Analysis, 4 (1969), 153-165.

3. M. Hayashi, Point derivations on commutative Banach algebras and estimates of the $A(X)$-metric norm, J. Math. Japan, 27 (1975), 24-42.

4. A. G. O'Farrell, Equiconvergence of derivations, Pacific J. Math., 53 (1974), 539-554. 5. — An isolated bounded point derivation, Proc. Amer. Math. Soc., 39 (1973), $559-562$.

6. J. Li-ming Wang, An approximate Taylor's theorem for $R(X)$, Math. Scand., 33 (1973), 343-358. 
7. T. W. Gamelin and J. Garnett, Pointwise bounded approximation and Dirichlet algebras, J. Functional Analysis, 8 (1971), 360-404.

8. A. G. O'Farrell, Analytic capacity, Hölder conditions, and $\tau$-spikes, Trans. Amer. Math. Soc., 196 (1974), 415-424.

Received September 15, 1975 and in revised form July 6, 1976.

IBARAKI UNIVERSITY

Current address: University of California, Los Angeles 


\section{PACIFIC JOURNAL OF MATHEMATICS}

EDITORS

RICHARD ARENS (Managing Editor)

University of California

Los Angeles, California 90024

R. A. BEAUMONT

University of Washington

Seattle, Washington 98105

J. DugundjI

Department of Mathematics

University of Southern California

Los Angeles, California 90007

D. Gilbarg and J. Milgram

Stanford University

Stanford, California 94305

\section{ASSOCIATE EDITORS}

E. F. BeCKENBACH

B. H. NeUMaNN

F. WOLF

K. YoSHIDA

\section{SUPPORTING INSTITUTIONS}

UNIVERSITY OF BRITISH COLUMBIA

CALIFORNIA INSTITUTE OF TECHNOLOGY

UNIVERSITY OF CALIFORNIA

MONTANA STATE UNIVERSITY

UNIVERSITY OF NEVADA

NEW MEXICO STATE UNIVERSITY

OREGON STATE UNIVERSITY

UNIVERSITY OF OREGON

OSAKA UNIVERSITY

\author{
UNIVERSITY OF SOUTHERN CALIFORNIA \\ STANFORD UNIVERSITY \\ UNIVERSITY OF TOKYO \\ UNIVERSITY OF UTAH \\ WASHINGTON STATE UNIVERSITY \\ UNIVERSITY OF WASHINGTON \\ AMERICAN MATHEMATICAL SOCIETY \\ NAVAL WEAPONS CENTER
}




\section{Pacific Journal of Mathematics \\ Vol. 67, No. $1 \quad$ January, 1976}

Gregory Wayne Brumfiel and John W. Morgan, Homotopy theoretic

consequences of $N$. Levitt's obstruction theory to transversality for

spherical fibrations . ................................ 1

Jacob Burbea, Total positivity of certain reproducing kernels ........... 101

Wai-Mee Ching, The structure of standard $C^{*}$-algebras and their

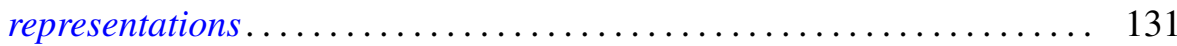

Satya Deo, The cohomological dimension of an $n$-manifold is $n+1 \ldots \ldots 155$

Masahiko Fujiwara and Masaki Sudo, Some forms of odd degree for which the Hasse principle fails ................................. 161

Mikihiro Hayashi, Smoothness of analytic functions at boundary points ... 171

Rebecca A. Herb, A uniqueness theorem for tempered invariant

eigendistributions ................................ 203

David Alan Legg, Orlicz space convergence of martingales of

Radon-Nikodým derivatives given a $\sigma$-lattice.................. 209

D. B. McAlister, v-prehomomorphisms on inverse semigroups......... 215

Bruno J. Mueller, Localization in fully bounded Noetherian rings . ........ 233

Donald J. Newman and A. R. Reddy, Rational approximation to $x^{n} \ldots \ldots .247$

Abraham Ziv, Inclusion relations between power methods of limitation.... 251 